\title{
The stability of developing pipe flow at high Reynolds number and the existence of nonlinear neutral centre modes
}

\author{
By ANDREW G. WALTON \\ Department of Mathematics, Imperial College London, South Kensington Campus, London \\ SW7 2AZ, UK
}

The high-Reynolds-number stability of unsteady pipe flow to axisymmetric disturbances is studied using asymptotic analysis. It is shown that as the disturbance amplitude is increased, nonlinear effects first become significant within the critical layer which moves away from the pipe wall as a result. It is found that the flow stabilizes once the basic profile has become sufficiently fully-developed. By tracing the nonlinear neutral curve back to earlier times, it is found that in addition to the wall mode, which arises from a classical upper branch linear stability analysis, there also exists a nonlinear neutral centre mode, governed primarily by inviscid dynamics. The centre mode problem is solved numerically and the results show the existence of a concentrated region of vorticity centered on or close to the pipe axis and propagating downstream at almost the maximum fluid velocity. The connection between this structure and the puffs and slugs of vorticity observed in experiments is discussed.

\section{Introduction}

\subsection{Background}

One of the first controlled experiments on fluid flow was Reynolds (1883) investigation into pipe flow transition. Well over a century later, some of his observations still lack a firm theoretical explanation, not least because of the fact that the basic fully-developed HagenPoiseuille flow (HPF) is thought to be theoretically stable to infinitesimal disturbances (see for example, the numerical calculations of Salwen, Cotton \& Grosch 1980). At a critical fluid speed Reynolds observed transient bursts of turbulence with these patches propagating along and out of the end of the pipe, to be replaced by a new turbulent region at the same location. Much later, an extensive series of investigations was carried out by Wygnanski \& Champagne (1973) and Wygnanski, Sokolov \& Friedman (1975). The intermittent turbulent regions were once again observed and this time denoted by the terms 'puff' and 'slug'. Slugs have a streamwise extent of many pipe diameters, fill the entire cross-section of the pipe, and are strongest near the pipe wall, from whence they are thought to originate. Puffs generally occur at lower Reynolds numbers and at higher levels of disturbance. They are concentrated near the pipe axis, and appear to be fairly unaffected by events occurring closer to the wall. The properties of puffs and slugs were investigated further in papers such as Bandyopadhyay (1986) and Nishi, Unsal, Durst \& Biswas (2008). Although in most of these experiments the perturbations are introduced in the inlet region, similar structures have been observed in fully-developed flow by Darbyshire \& Mullin (1995) and van Doorne \& Westerweel (2008), among others. Again, 
most of the disturbances introduced in experiments have been axially nonsymmetric, but the experiments of Leite (1959) and Fox, Lessen \& Bhat (1968) show that turbulent structures of the form described above can also arise as a result of strong axisymmetric input.

Over the years, many direct numerical simulations of the unsteady Navier-Stokes equations have been performed to see whether similar flow structures emerge to those observed in experiments. This approach, aided by the considerable computing power available to modern researchers, has been successful in as much as it has confirmed that the incompressible equations do indeed capture the necessary physics to explain pipe flow transition. For example, O'Sullivan \& Breuer (1994), Shan, Ma, Zhang \& Nieuwstadt (1999), Priymak \& Miyazaki (2004) and Duguet, Willis \& Kerswell (2010) are all able to reproduce some of the features of puffs and slugs in their numerical experiments. By directly seeking travelling-wave solutions to a forced version of the Navier-Stokes equations, and then gradually reducing the forcing, Faisst \& Eckhardt (2003) and Wedin \& Kerswell (2004) have found that such solutions exist, as an alternative to HPF, for a range of azimuthal wavenumbers. Axisymmetric travelling solutions have so far not been found by this approach.

Fowler \& Howell (2003) adopted a heuristic approach which has yielded some interesting results. They proposed a simple model which possesses some of the essential properties of pipe flow (e.g. linear stability at all Reynolds numbers and nonlinear instability above a critical value) and were able to show that this model predicts some of the features of turbulent puffs and slugs. The difficulty with such a model is that it is inevitably fine-tuned using empirical data, and its connection to the Navier-Stokes equations is not entirely clear.

Another theoretical approach that explains some aspects of the transition process is to assume that the disturbances are small and appeal to linear theory. Despite the fact that ultimately all small disturbances to HPF are damped, there can be strong initial growth due to the non-normal structure of the linear stability operator. This approach is discussed in detail in Schmid \& Henningson (2000) and it leads to predictions of large transient growth rates for disturbances with an azimuthal wavenumber $N$ of unity. However, for axisymmetric modes $(N=0)$, transient growth only occurs for sufficiently large values of $\alpha R$ where $R$ is Reynolds number (based on pipe radius and centreline velocity), and $\alpha$ axial wavenumber.

An alternative approach to trying to understand the possible routes to transition in pipe flow is to consider a base state that is still evolving, either in space or time. One of the first numerical studies in this area was by Tatsumi (1952a,b), who studied the linear stability of spatially-developing pipe flow using a model base profile and found the flow to be linearly unstable in the developing region at Reynolds numbers of about 20,000. Huang and Chen (1974a,b) repeated these calculations using a more realistic model profile and found a critical Reynolds number of close to 40,000. Small-disturbance experiments were then performed by Sarpkaya (1975) which also found instability in the inlet region of the pipe but at a considerably lower Reynolds number (typically around 8,000 ) for both axisymmetric and nonsymmetric disturbances. Later, Bandyopadhyay \& Walton (1989) performed experiments on the instability of the inlet flow to larger disturbances and found a slug-type transition with a critical Reynolds number of around 3,000 .

In this paper we will revisit the stability of developing pipe flow and use asymptotic analysis to divide the flow into various regions, describe the dominant physical processes in each layer, solve the equations in each and then match them together. Almost inevitably, because of the inherent nonlinearity present in any suitable disturbance, at 
least some of the regions will be governed by equations which require a numerical solution: typically however, the numerical tasks involved are much less onerous than those involved in a full Navier-Stokes simulation. Of course, in order to perform asymptotic analysis we need a suitable large parameter to be present, and in the pipe flow transition problem, since the critical Reynolds number is only several thousand, it is not clear that a high Reynolds number assumption is appropriate, or indeed will yield fruitful results. The usefulness of such an approach was demonstrated by Smith \& Bodonyi (1982) (see also minor corrections in Walton 2004). Drawing on ideas they had developed for nonlinear boundary-layer instability, they proposed a disturbance structure for HPF which takes the form of an inviscid travelling wave across the majority of the pipe. In the thin critical layer surrounding the location where the disturbance wavespeed matches the basic flow velocity, the disturbance is governed by nonlinear dynamics and gives rise to an asymptotically small phase shift across the layer, as opposed to the $O(1)$ phase shift of linear theory (see, for example, Stuart 1963). This phase shift is then reduced to zero by viscous processes within the wall layer provided the disturbance amplitude $A$ is dependent in a specific way on the wavenumbers $\alpha$ and $N$. One of the features of this work was the fact that very little numerical computation was necessary in order to determine $A(\alpha, N)$ and a key result was that this travelling wave structure only exists if $N=1$. Motivated by this work, Walton (2002) showed, by considering impulsively-started pipe flow, that the Smith \& Bodonyi neutral criteria arise from linear upper-branch stability properties as the disturbance size is increased. This study also showed that modes with $N>1$ can be supported up to a finite value of the time parameter $\widetilde{t}$ say, with only the $N=1$ mode surviving once the basic flow becomes fully-developed.

Both of these studies concentrate on nonsymmetric modes $N \neq 0$, but the experiments mentioned earlier show that the same route to transition occurs when the imposed disturbances are axially symmetric. Our original motivation for the study we describe here was to investigate whether a similar analysis to Walton (2002) could be performed for $N=0$. If so, it would be interesting to know whether such modes disappear at a finite time after the fluid has been set into motion or whether they can survive as nonlinear disturbances to HPF. We begin by analyzing the linear stability of the developing flow just after a pressure gradient has been applied. At high Reynolds number there exists an asymptotic structure in which the wavespeed, although small, is sufficiently large that the critical layer and wall layer are distinct. This is the so-called 'upper branch'. The linear and weakly nonlinear upper branch stability results are discussed briefly in sections 2 and 3 . We find that there are many similarities to the nonsymmetric situation, but a number of new features also arise. It is explained in section 4 how the structure remains intact into the 'almost-developed' region where $\widetilde{t} \sim O(1)$, but now the disturbances are much larger than their nonsymmetric counterparts. We find that there is a 'cut-off' at a finite value of $\widetilde{t}$ as in the $N \neq 1$ asymmetric case discussed above, but as the value of $\widetilde{t}$ is decreased back towards zero a second solution emerges in which the disturbance remains nonlinear while the critical layer moves towards the pipe centre. The new mode structure is very similar to the nonlinear 'slug' structure proposed by Smith, Doorly \& Rothmayer (1990), and the simplifications due to axisymmetry allow us to compute solutions to the problem: these are analyzed in some detail in section 5. A discussion of all the work reported here is presented in section 6 .

\subsection{The governing equations and basic flow}

We use cylindrical polar coordinates $(a x, a r, \theta)$ with origin at the pipe entrance, where $a x$, ar represent the axial and radial coordinates respectively. Since the flow is assumed to be axisymmetric with no swirl, there is no component of velocity in the azimuthal 
$(\theta)$ direction and no dependence upon $\theta$ throughout. The $(x, r)$ velocity components are written as $\left(g a^{2} / \rho \nu\right)(u, v)$, where $4 g$ is the constant axial pressure gradient to be applied to the pipe, $a$ is its radius, and the constants $\rho$ and $\nu$ are the density and kinematic viscosity of the incompressible fluid. The pressure scaling is $\left(g^{2} a^{4} / \rho \nu^{2}\right) p$, and the time, based on fluid velocity and pipe radius, is written in the form $(\rho \nu / g a) t$. It is convenient for what follows later to also define a viscous time $\left(a^{2} / \nu\right) \widetilde{t}$. These scalings enable us to write the governing axisymmetric, unsteady Navier-Stokes equations in the non-dimensional form:

$$
\begin{gathered}
\frac{\partial u}{\partial x}+\frac{\partial v}{\partial r}+\frac{v}{r}=0 \\
\frac{\partial u}{\partial t}+u \frac{\partial u}{\partial x}+v \frac{\partial u}{\partial r}=-\frac{\partial p}{\partial x}+\frac{1}{R}\left(\nabla^{2} u-\frac{\partial u}{\partial \widetilde{t}}\right) \\
\frac{\partial v}{\partial t}+u \frac{\partial v}{\partial x}+v \frac{\partial v}{\partial r}=-\frac{\partial p}{\partial r}+\frac{1}{R}\left(\nabla^{2} v-\frac{v}{r^{2}}-\frac{\partial v}{\partial \widetilde{t}}\right)
\end{gathered}
$$

with $\nabla^{2} \equiv \partial^{2} / \partial x^{2}+\partial^{2} / \partial r^{2}+r^{-1} \partial / \partial r$, and Reynolds number $R$ defined by

$$
R=\frac{g a^{3}}{\rho \nu^{2}}
$$

The basic viscous flow is taken to be parallel and unsteady, with $u=u_{0}(r, \widetilde{t}), v=0$, and in non-dimensional terms, the pressure gradient $\partial p / \partial x=-4 / R$ is applied to the pipe at time $\widetilde{t}=0$ say, setting the fluid into motion. The appropriate exact solution of (1.1) has the well-known form (e.g. Szymanski 1932, Batchelor 1967):

$$
u_{0}(r, \widetilde{t})=1-r^{2}-8 \sum_{n=1}^{\infty} \frac{J_{0}\left(j_{o n} r\right)}{j_{o n}^{3} J_{1}\left(j_{o n}\right)} \exp \left(-j_{o n}^{2} \widetilde{t}\right)
$$

where $j_{o n}$ is the $n$th zero of the Bessel function $J_{0}$. For values of viscous time such that $\widetilde{t} \ll 1$, it can easily be shown that the solution acquires the multi-structured form

$$
u_{0}(r, \widetilde{t}) \sim \begin{cases}4 \widetilde{t} & r \text { of } O(1) \\ 4 \widetilde{t} u_{B}(\eta) & r=1-\widetilde{t}^{1 / 2} \eta\end{cases}
$$

to leading order, with

$$
u_{B}(\eta)=\left(1+\frac{1}{2} \eta^{2}\right) \operatorname{erf}\left(\frac{1}{2} \eta\right)-\frac{1}{2} \eta^{2}+\frac{\eta}{\sqrt{\pi}} \exp \left(-\eta^{2} / 4\right) .
$$

One approach to analyzing the stability of this flow is to formulate an Orr-Sommerfeldtype problem and calculate, for given $\widetilde{t}$, curves of constant growth rate, including a neutral curve. Calculations carried out by the present author indicate, not surprisingly, the existence of a neutral curve for $\widetilde{t} \ll 1$ which is broadly similar to that for Blasius boundarylayer flow. As $\tilde{t}$ is increased the neutral curve retreats to infinity, in accordance with the well-known result that HPF is linearly stable, as mentioned earlier. Although this approach yields some interesting results, it is of course fundamentally flawed as in general the timescale associated with the disturbance frequency is comparable to that over which the basic flow is evolving. An Orr-Sommerfeld approach is therefore not mathematically consistent as implicit in this formulation is the neglect of the time derivatives of the basic flow. In general then, the neutral curves generated in this way do not represent valid solutions of the stability problem. However, the large Reynolds number asymptotes of the two branches of the neutral curve are the result of a mathematically consistent procedure, as on these branches the disturbance timescale is asymptotically smaller (in 
terms of the Reynolds number) than that of the basic flow, and therefore time derivatives of the basic flow can be neglected to leading order. Moreover, these asymptotic structures remain essentially intact as the disturbance size is increased, and so this high Reynolds number approach allows us to incorporate the effects of nonlinearity in a self-consistent manner. In Walton (2002) the mode structure on the upper branch of the weakly nonlinear neutral curve was analyzed at large Reynolds number for nonsymmetric disturbances proportional to $\exp (\mathrm{i} N \theta)$. There it was found that for $N=1$ the asymptotic structure develops into the travelling-wave structure for HPF proposed by Smith \& Bodonyi (1982), with the time dependence of the basic flow having a purely parametric effect on the nonlinear modes. As mentioned earlier, modes corresponding to larger values of $N$ experienced cut-off at finite values of $\widetilde{t}$ and therefore are not directly relevant to the stability of HPF. In this paper we once again focus on how the upper branch stability structure alters as the disturbance size is increased but we now concentrate exclusively on axisymmetric modes.

\section{The asymptotic upper branch structure in the linear regime}

We start by considering the linear stability of the flow for $\tilde{t} \ll 1$ when the two-tier form (1.4) is appropriate. If we superimpose a small disturbance $(\widehat{u}, \widehat{v}, \widehat{p})$ on the basic flow $u_{0}$, then the disturbance equations are:

$$
\begin{gathered}
\frac{\partial \widehat{u}}{\partial x}+\frac{\partial \widehat{v}}{\partial r}+\frac{\widehat{v}}{r}=0 \\
\frac{\partial \widehat{u}}{\partial t}+u_{0} \frac{\partial \widehat{u}}{\partial x}+\widehat{v} \frac{\partial u_{0}}{\partial r}=-\frac{\partial \widehat{p}}{\partial x}+\frac{1}{R}\left(\nabla^{2} \widehat{u}-\frac{\partial \widehat{u}}{\partial \widetilde{t}}\right) \\
\frac{\partial \widehat{v}}{\partial t}+u_{0} \frac{\partial \widehat{v}}{\partial x}=-\frac{\partial \widehat{p}}{\partial r}+\frac{1}{R}\left(\nabla^{2} \widehat{v}-\frac{\widehat{v}}{r^{2}}-\frac{\partial \widehat{v}}{\partial \widetilde{t}}\right) .
\end{gathered}
$$

We now express the disturbance in the wave-like form

$$
(\widehat{u}, \widehat{v}, \widehat{p})=\mathfrak{R}((F(r, \widetilde{t}), \mathrm{i} G(r, \widetilde{t}), P(r, \widetilde{t})) \exp (\mathrm{i} \xi)),
$$

where $\mathfrak{R}$ denotes 'the real part', $\xi=\alpha(x-c t)$ and we seek neutral solutions with $\alpha$ and $c$ real. It is shown in Walton (2002) that, at least as far as nonsymmetric modes are concerned, curvature effects first enter the upper branch stability structure at viscous times of $O\left(R^{-2 / 9}\right)$ after the fluid is set into motion. This occurs because at this time, the radial velocity in the wall layer has grown to a sufficient size to balance the curvatureinduced phase shift from the critical layer. Thus we set

$$
\tilde{t}=R^{-2 / 9} t_{0}
$$

and will show subsequently that the upper branch structure is only self-consistent provided $t_{0} \ll O\left(R^{2 / 9}\right)$. Derivatives with respect to time can be expressed as

$$
\frac{\partial}{\partial t}=-\mathrm{i} \alpha c ; \quad R^{-1} \frac{\partial}{\partial \widetilde{t}}=R^{-7 / 9} \frac{\partial}{\partial t_{0}}
$$

with $t_{0}$ having a purely parametric effect to the order we work provided $\alpha c \gg R^{-7 / 9} t_{0}^{-1}$, which we will show is indeed the case in this linear regime provided $R$ is sufficiently large. In other words, in the high Reynolds number limit, the timescale associated with the wave variation will be shown to be much faster than the viscous timescale associated with the basic flow. There are five main flow regions all possessing similar properties to their counterparts in the structure for nonsymmetric modes investigated by Walton 
(2002). A sketch of the structure is shown in figure 1(a). We present only brief details of the scalings and matching procedures here, with the reader referred to the last-named paper for more details.

(i) Region I. This region occupies the majority of the pipe, and the basic flow $u_{0} \sim$ $4 R^{-2 / 9} t_{0}$ to leading order in view of the upper tier of (1.4) and (2.1). The pressure disturbance satisfies a form of Laplace's equation and the solution which is finite on the pipe axis has

$$
P=\frac{I_{0}\left(\alpha_{0} r\right)}{I_{0}\left(\alpha_{0}\right)},
$$

where the $O(1)$ constant $\alpha_{0}$ is the leading order part of the wavenumber, $I_{0}$ is a modified Bessel function and we have taken $P=1$ at $r=1$ as our normalization condition.

(ii) Region II. Here the basic flow is given by the similarity form in the lower tier of (1.4) and the radial scaling is therefore

$$
r=1-R^{-1 / 9} t_{0}^{1 / 2} \eta .
$$

The pressure expands in the form

$$
P=1+R^{-1 / 9} t_{0}^{1 / 2} p_{1}(\eta)+\cdots,
$$

and the solution which matches appropriately to that in region I above has

$$
p_{1}^{\prime}=-\alpha_{0} \frac{I_{0}^{\prime}\left(\alpha_{0}\right)}{I_{0}\left(\alpha_{0}\right)}\left(u_{B}(\eta)\right)^{2} .
$$

(iii) Region III. In this region the basic flow is of near-wall shear form deduced from the behaviour

$$
u_{B}(\eta) \sim \lambda_{0} \eta+\lambda_{1} \eta^{2} \text { as } \eta \rightarrow 0 \text { with } \lambda_{0}=\frac{2}{\sqrt{\pi}}, \lambda_{1}=-\frac{1}{2},
$$

which follows from (1.5). The correct scalings for the region are determined by introducing the effects of streamwise diffusion into the equation governing the correction to the wall pressure. The appropriate expansions are

$$
\begin{gathered}
r=1-R^{-2 / 9} t_{0} Y, \\
P=1+R^{-4 / 9} t_{0}^{2} \widehat{p}_{1}(\zeta)+R^{-5 / 9} t_{0}{ }^{5 / 2} \widehat{p}_{2}(\zeta)+\cdots, \\
c=4 R^{-1 / 3} t_{0}^{3 / 2} \bar{c}_{0}+\cdots,
\end{gathered}
$$

with the coordinate $\zeta \equiv \lambda_{0} Y-\bar{c}_{0}$ describing radial variations. The leading-order pressure perturbation solution which matches to region II is found to be such that

$$
\widehat{p}_{1}^{\prime}=-\frac{\alpha_{0}}{\lambda_{0}} \frac{I_{0}^{\prime}\left(\alpha_{0}\right)}{I_{0}\left(\alpha_{0}\right)} \zeta^{2}-\frac{\alpha_{0}^{2}}{\lambda_{0}^{2}} \zeta
$$

and upon applying the tangential flow condition that $\widehat{p}_{1}^{\prime} \rightarrow 0$ as $Y \rightarrow 0$ we obtain the condition

$$
\bar{c}_{0} \lambda_{0} I_{0}^{\prime}\left(\alpha_{0}\right)=\alpha_{0} I_{0}\left(\alpha_{0}\right)
$$

which provides a first relation between $\alpha_{0}$ and $\bar{c}_{0}$. To find a second relation we proceed to higher order where we find that

$$
\widehat{p}_{2}^{\prime}(\zeta)=(\text { terms regular in } \zeta)-\frac{2 \lambda_{1} \alpha_{0}^{2}}{\lambda_{0}^{4}} \zeta^{2} \ln \zeta, \quad(\zeta>0) .
$$

The irregular behaviour of the second term on the right-hand-side of (2.6) implies the 
existence of a viscous critical layer (region $I V$, say) at $\zeta=0$. Applying the classical $-\pi$ phase shift (Lin 1955, Stuart 1963, Reid 1965) we have that terms involving $\ln \zeta$ for $\zeta>0$ are replaced by $\ln |\zeta|-i \pi$ for $\zeta<0$. Thus, as far as the radial velocity is concerned, a $\cos \xi$ component, $\widehat{v}_{c}$ say, is induced upon crossing the critical layer, and as the wall is approached we find

$$
\widehat{v}_{c} \rightarrow-\frac{\pi \lambda_{1} \bar{c}_{0} \alpha_{0}}{2 \lambda_{0}^{3}} \text { as } r \rightarrow 1,
$$

upon use of the radial scaling $(2.3 a)$.

(iv) Region $V$. This is the viscous wall layer where the disturbance velocities are reduced to zero to satisfy the no-slip condition. The radial velocity asymptotically far from the wall must also match to the result (2.7). In this layer the disturbance frequency $\alpha c \sim$ $O\left(R^{-1 / 3} t_{0}^{3 / 2} \alpha_{0}\right)$ from $(2.3 c)$ and is in balance with the viscous operator $R^{-1} \partial^{2} / \partial r^{2}$. As a consequence, the appropriate radial scaling is

$$
r=1-\alpha_{0}^{-1 / 2} R^{-1 / 3} t_{0}^{-3 / 4} \bar{Y},
$$

while the velocities and pressure expand as

$$
\begin{gathered}
\widehat{u}=R^{1 / 3} t_{0}{ }^{-3 / 2} \alpha_{0}^{-1} \mathfrak{R}\left(\bar{u}(\bar{Y}) \mathrm{e}^{\mathrm{i} \xi}\right)+\cdots, \\
\widehat{v}=-t_{0}{ }^{-9 / 4} \alpha_{0}^{-3 / 2} \mathfrak{R}\left(i \bar{v}(\bar{Y}) \mathrm{e}^{\mathrm{i} \xi}\right)+\cdots, \\
\widehat{p}=\cos \xi+\cdots .
\end{gathered}
$$

The solutions of the resulting viscous balances that satisfy no-slip at the wall are

$$
\begin{gathered}
\bar{u}=\frac{\alpha_{0}}{4 \bar{c}_{0}}(1-\exp (-m \bar{Y})) \\
\bar{v}=-\frac{\alpha_{0}^{2}}{4 \bar{c}_{0}}\left(\bar{Y}-\frac{1}{m}(1-\exp (-m \bar{Y}))\right),
\end{gathered}
$$

with $m \equiv\left(2 \bar{c}_{0}\right)^{1 / 2}(1-\mathrm{i})$. From this solution it follows that

$$
\widehat{v}_{c} \rightarrow 2^{-7 / 2} t_{0}^{-9 / 4} \bar{c}_{0}^{-3 / 2} \alpha_{0}^{1 / 2} \text { as } \bar{Y} \rightarrow \infty .
$$

This must be consistent with the result (2.7) and leads to the relation

$$
\frac{1}{2^{7 / 2} t_{0}^{9 / 4} \alpha_{0}^{3 / 2} \bar{c}_{0}^{3 / 2}}=-\frac{\pi \lambda_{1} \bar{c}_{0}}{2 \lambda_{0}^{3} \alpha_{0}} .
$$

Eliminating $\bar{c}_{0}$ between the eigenrelations (2.5) and (2.11) we obtain the result

$$
\alpha_{0}^{6 / 5} \frac{I_{0}\left(\alpha_{0}\right)}{I_{0}^{\prime}\left(\alpha_{0}\right)}=\frac{\lambda_{0}^{11 / 5}}{2 \pi^{2 / 5}\left(-\lambda_{1}\right)^{2 / 5} t_{0}^{9 / 10}},
$$

giving $\alpha_{0}$ as a function of $t_{0}$, which should be compared with the analogous asymmetric result obtained by Walton (2002). As mentioned earlier, the effect of time evolution is observed as a parametric effect in (2.12).

Our main interest here is how the neutral criteria alter as the basic flow develops. Letting $t_{0} \rightarrow \infty$ in $(2.12)$ we obtain

$$
\alpha_{0} \sim \frac{\lambda_{0}^{11}}{2^{10} \pi^{2} \lambda_{1}^{2}} t_{0}^{-9 / 2}, c \sim\left(8 / \lambda_{0}\right) R^{-1 / 3} t_{0}^{3 / 2},
$$

using the formula $I_{0}\left(\alpha_{0}\right) / I_{0}^{\prime}\left(\alpha_{0}\right) \sim 2 / \alpha_{0}$ as $\alpha_{0} \rightarrow 0$. It is worth mentioning that the asymptotic form for $\alpha_{0}$ cannot simply be found by setting $N=0$ in the analogous 

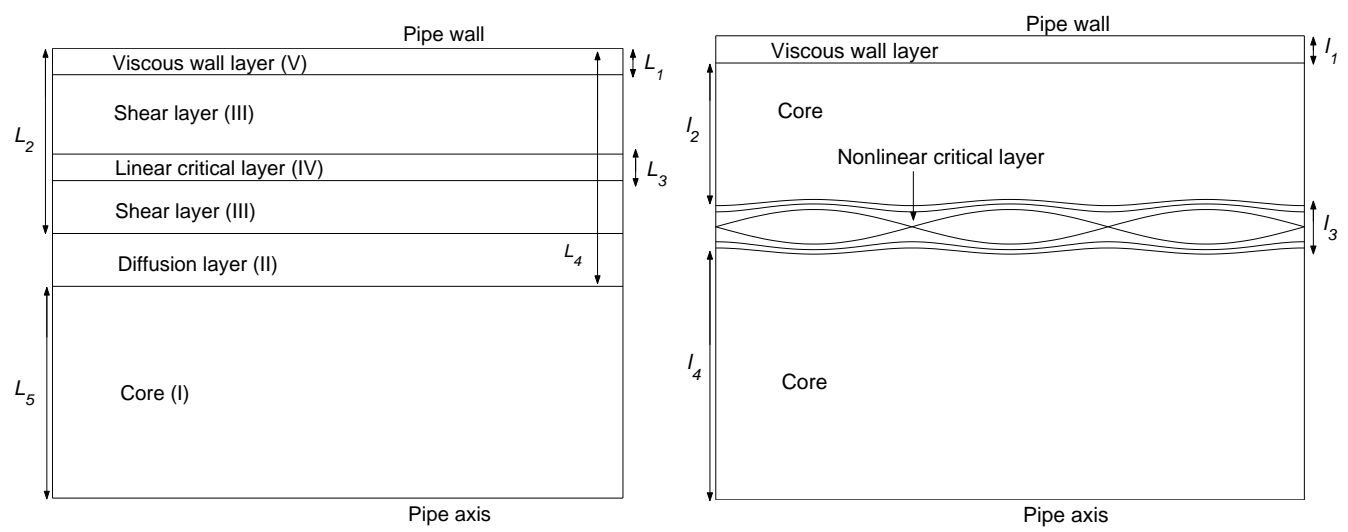

Figure 1. (a) The five-tier linear upper branch structure for developing pipe flow for viscous times $\widetilde{t} \ll 1$. The thicknesses of the various regions in terms of $\widetilde{t}$ are $L_{1} \sim \widetilde{t}^{3 / 2}, L_{2} \sim \widetilde{t}, L_{3} \sim \widetilde{t}^{4 / 3}$, $L_{4} \sim \widetilde{t}^{1 / 2}, L_{5} \sim O(1)$. (b) The nonlinear upper branch structure for $\widetilde{t} \sim O(1)$ and $\widetilde{\alpha} \equiv \alpha R \gg 1$. In terms of $\widetilde{\alpha}$, the thicknesses of the various regions are $1_{1} \sim \widetilde{\alpha}^{-1 / 2}, l_{2} \sim O(1), l_{3} \sim \widetilde{\alpha}^{-1 / 6}$, $l_{4} \sim O(1)$.

asymmetric result. Results (2.13) remain valid provided $\alpha c \gg R^{-7 / 9} t_{0}^{-1}$ as remarked earlier. We therefore conclude that the upper branch structure stays intact until

$$
\widetilde{t}=R^{-2 / 9} t_{0} \sim O(1) .
$$

At this critical viscous time the locally neutral wavespeed becomes $O(1)$, the wavenumber $O\left(R^{-1}\right)$, the two-tier structure for the basic flow merges into one, the two timescales coincide and all the asymptotic regions analyzed above become comparable with the pipe diameter. The multi-tier structure for $\widetilde{t} \ll 1$ with scalings in terms of $\widetilde{t}$ is shown in figure $1(\mathrm{a})$. In view of the breakdown of the structure, an asymptotic approach to the linear stability problem is therefore not possible at $O(1)$ values of $\widetilde{t}$. However we will see later that an asymptotic structure still exists at $O(1)$ times for enhanced disturbances. To see why this is the case it is instructive to examine the critical layer dynamics in more detail as this is where the main changes to the flow take place as a result of an increased disturbance size.

\section{The upper branch structure in the presence of weak nonlinearity}

The physical balance within the critical layer arises from the comparable sizes of the inertial and viscous operators $\left(u_{0}-c\right) \partial / \partial x, R^{-1} \partial^{2} / \partial r^{2}$ and leads to a critical layer thickness $\Delta \sim \alpha_{0}^{-1 / 3} t_{0}^{-1 / 6} R^{-8 / 27}$ as in Walton (2002). Here, the axial perturbation is of size $\delta R^{2 / 9} t_{0}^{-1}$, where $\delta$ is the (small) arbitrary amplitude of the pressure disturbance. The first major change to the critical-layer properties, as in the nonsymmetric case, arises when the axial perturbation becomes as large as the curvature contribution (of order $\Delta^{2}$ ) to the basic flow. A new stage is therefore reached when $\delta \sim R^{-22 / 27} t_{0}^{2 / 3} \alpha_{0}^{-2 / 3}$. We set

$$
\delta=R^{-22 / 27} t_{0}^{2 / 3} \alpha_{0}^{-2 / 3} \widehat{A}
$$

and investigate the dynamics of the resultant weakly nonlinear critical layer. If we define an order one variable $\widehat{\zeta}$ to describe local radial variations within the critical layer then 
in terms of the original variables we have

$$
r=1-R^{-2 / 9} t_{0} \frac{\bar{c}_{0}}{\lambda_{0}}-R^{-8 / 27} t_{0}^{-1 / 6} \alpha_{0}^{-1 / 3} \frac{\widehat{\zeta}}{\lambda_{0}} .
$$

Using a streamfunction $\psi$, the motion is governed at leading order by the momentum balance

$$
4 \widehat{\zeta} \frac{\partial^{3} \psi}{\partial \xi \partial \widehat{\zeta}^{2}}+\frac{\widehat{A}}{4} \sin \xi \frac{\partial^{3} \psi}{\partial \widehat{\zeta}^{3}}=\lambda_{0}^{2} \frac{\partial^{4} \psi}{\partial \widehat{\zeta}^{4}},
$$

with the matching conditions to region III being

$$
\begin{array}{r}
\frac{\partial \psi}{\partial \widehat{\zeta}} \sim \frac{4 \lambda_{1}}{\lambda_{0}^{2}} \widehat{\zeta}^{2}+\widehat{A}^{1 / 2} \widehat{\lambda}^{+} \widehat{\zeta}+\widehat{A} \frac{\lambda_{1}}{2 \lambda_{0}^{2}}(\ln \widehat{\zeta})(\cos \xi) \text { as } \widehat{\zeta} \rightarrow+\infty \\
\frac{\partial \psi}{\partial \widehat{\zeta}} \sim \frac{4 \lambda_{1}}{\lambda_{0}^{2}} \widehat{\zeta}^{2}+\widehat{A}^{1 / 2} \widehat{\lambda}^{-} \widehat{\zeta}+\widehat{A} \frac{\lambda_{1}}{2 \lambda_{0}^{2}}(\ln |\widehat{\zeta}| \cos \xi-J(\xi)) \text { as } \widehat{\zeta} \rightarrow-\infty
\end{array}
$$

Here, the velocity jump $J(\xi)$ is determined as part of the solution, while the constant $\widehat{\lambda}^{+}-\widehat{\lambda}^{-}$represents the jump in vorticity across the layer. The phase shift $\phi$ is no longer equal to its linear value of $-\pi$, but can be derived from the velocity jump via

$$
\phi=\frac{1}{\pi} \int_{0}^{2 \pi} J(\xi) \sin \xi \mathrm{d} \xi
$$

The vorticity jump and phase shift can be related by integrating the critical layer equation (3.2) and applying the matching conditions (3.3). We find that

$$
\phi=\frac{16 \lambda_{0}^{4}}{\lambda_{1}}\left(\widehat{\lambda}^{+}-\widehat{\lambda}^{-}\right) \widehat{A}^{-3 / 2}
$$

The large-time wavenumber result (2.13a) is unaltered from its linear form except for the replacement of the classical phase shift $-\pi$ by $\phi$, i.e. we now have

$$
\alpha_{0} \sim \frac{\lambda_{0}^{11}}{2^{10} \lambda_{1}^{2}} \frac{t_{0}^{-9 / 2}}{\phi^{2}} \text { as } t_{0} \rightarrow \infty
$$

We are particularly interested here in the strong nonlinearity limit $\widehat{A} \gg 1$. In this limit it is easy to show from analysis of (3.2) that the vorticity jump

$$
\left(\widehat{\lambda}^{+}-\widehat{\lambda}^{-}\right) \rightarrow \frac{\lambda_{1} C^{(1)}}{\lambda_{0}^{2}} \text { as } \widehat{A} \rightarrow \infty
$$

where $C^{(1)} \simeq-5.516$ (Smith \& Bodonyi 1982). Thus in this large amplitude limit we obtain $\phi \sim 16 \lambda_{0}^{2} C^{(1)} \widehat{A}^{-3 / 2}$, and hence

$$
\alpha_{0} \sim R^{-1} \widetilde{\alpha}, \text { with } \widetilde{\alpha}=\frac{\lambda_{0}^{7}}{2^{18} \lambda_{1}^{2}\left(C^{(1)}\right)^{2}} \widehat{A}^{3} \widetilde{t}^{-9 / 2} \gg 1 .
$$

In terms of the scaled wavenumber $\widetilde{\alpha}$ and viscous time $\widetilde{t}$, the corresponding forms for the disturbance amplitude, phase shift and wavespeed are

$$
\delta \sim \widetilde{\alpha}^{-1 / 3} \frac{2^{6}\left(\lambda_{1} C^{(1)}\right)^{2 / 3}}{\lambda_{0}^{7 / 3}} \widetilde{t}^{13 / 6}, \phi \sim \widetilde{\alpha}^{-1 / 2} \frac{\lambda_{0}^{11 / 2}}{2^{5} \lambda_{1}} \widetilde{t}^{-9 / 4}, \quad c \sim \frac{8}{\lambda_{0}} \widetilde{t}^{3 / 2}
$$

as $\tilde{t} \rightarrow 0$, from $(3.1),(3.7),(3.4),(2.13)$. It is worth noting that the constants of proportionality here differ from those obtained for nonsymmetric modes by Walton (2002). 
The result (3.7) is the key to further analysis: it shows that the disturbance wavelength is decreased by nonlinearity. In particular this means that even at viscous times of $O(1)$, when the basic flow is of the 'almost-developed' form (1.3), the disturbance wavelength is smaller than $O(R)$ and hence it may still be possible to analyze the disturbance behaviour in the strongly nonlinear regime without recourse to full numerical simulation.

\section{Neutral stability for enhanced disturbances: strongly nonlinear analysis}

The analysis of the weakly nonlinear critical layer suggests that in the almost-developed regime where $\tilde{t} \sim O(1)$, there should be a travelling wave structure with wavenumber $R^{-1} \widetilde{\alpha}$ and $\widetilde{\alpha} \gg 1$. In addition the pressure amplitude is predicted to be $O\left(\widetilde{\alpha}^{-1 / 3}\right)$, independent of Reynolds number (and larger than the nonsymmetric prediction in Walton 2002 ), while the wavespeed is $O(1)$. A nonlinear critical layer is anticipated, across which a small $O\left(\widetilde{\alpha}^{-1 / 2}\right)$ phase shift is induced. In order to investigate this structure we employ the axial scaling

$$
\frac{\partial}{\partial x}=R^{-1} \frac{\partial}{\partial \widetilde{x}}
$$

and retain the viscous time scale of the previous sections. The appropriate velocity and pressure scalings are

$$
\begin{aligned}
u & =\widetilde{u}(r, \widetilde{x}, \widetilde{t})+\cdots, \quad v=R^{-1} \widetilde{v}(r, \widetilde{x}, \widetilde{t})+\cdots \\
p & =\widetilde{p}(\widetilde{x}, \widetilde{t})-R^{-1} 4 x+R^{-2} \widetilde{p}_{R}(r, \widetilde{x}, \widetilde{t})+\cdots
\end{aligned}
$$

Substitution of these expansions into the Navier-Stokes equations (1.1) leads to the following balances from the continuity and axial momentum equations:

$$
\frac{\partial \widetilde{u}}{\partial \widetilde{x}}+\frac{\partial \widetilde{v}}{\partial r}+\frac{\widetilde{v}}{r}=0, \frac{\partial \widetilde{u}}{\partial \widetilde{t}}+\widetilde{u} \frac{\partial \widetilde{u}}{\partial \widetilde{x}}+\widetilde{v} \frac{\partial \widetilde{u}}{\partial r}=4-\frac{\partial \widetilde{p}}{\partial \widetilde{x}}+\frac{\partial^{2} \widetilde{u}}{\partial r^{2}}+\frac{1}{r} \frac{\partial \widetilde{u}}{\partial r}
$$

in which the Reynolds number has been scaled out. In addition, the radial momentum balance reduces at leading order to:

$$
\frac{\partial \widetilde{v}}{\partial \widetilde{t}}+\widetilde{u} \frac{\partial \widetilde{v}}{\partial \widetilde{x}}+\widetilde{v} \frac{\partial \widetilde{v}}{\partial r}=-\frac{\partial \widetilde{p}_{R}}{\partial r}+\frac{\partial^{2} \widetilde{v}}{\partial r^{2}}+\frac{1}{r} \frac{\partial \widetilde{v}}{\partial r}-\frac{\widetilde{v}}{r^{2}}
$$

We note that the system uncouples so that we need only consider equations (4.1), (which we will refer to as the reduced Navier-Stokes (RNS) equations) with (4.2) simply determining the higher-order pressure $\widetilde{p}_{R}$. In order to take account of the $\widetilde{\alpha}$-dependence, we seek disturbances proportional to

$$
\exp i \widetilde{\xi}, \quad \widetilde{\xi} \equiv \widetilde{\alpha}(\widetilde{x}-c \widetilde{t}),
$$

with $\widetilde{\alpha} \gg 1$, in which the pressure perturbation has amplitude

$$
\widetilde{\alpha}^{-1 / 3} A_{0}(\widetilde{t})
$$

in view of the form for $\delta$ in (3.8). The largeness of the scaled wavenumber here means that the timescale associated with the wave is still faster than that of the basic flow. The wavespeed is written as

$$
c=c_{0}+O\left(\widetilde{\alpha}^{-1 / 2}\right),
$$

with $c_{0}$ to be determined as part of the solution, while the basic flow is given by (1.3). For $\tilde{t}$ of $O(1)$, the stability structure consists of three main regions (figure $1 \mathrm{~b}$ ). There is an inviscid region, occupying the majority of the pipe, and a viscous wall layer, with these 
regions very similar in nature to regions $\mathrm{I}$ and $\mathrm{V}$ of the linear upper branch structure. In addition, with the wavespeed now being $O(1)$, the critical layer has moved away from the wall. We will consider each of these regions in turn. Their properties are similar to the Smith \& Bodonyi (1982) structure for HPF, but some new features arise because of the axisymmetric nature of the disturbances.

\subsection{The inviscid core region}

Since the pressure amplitude is already known, the velocity perturbations can also be determined, and the appropriate expansions are:

$$
\begin{array}{r}
\widetilde{u}=u_{0}(r, \widetilde{t})+\widetilde{\alpha}^{-1 / 6} \widetilde{u}_{1 M}(r, \widetilde{t})+\widetilde{\alpha}^{-1 / 3}\left(\widetilde{u}_{2}(r, \widetilde{x}, \widetilde{t})\right. \\
\left.\quad+\widetilde{u}_{2 M}(r, \widetilde{t})\right)+\cdots+\widetilde{\alpha}^{-5 / 6} \widetilde{u}_{5}(r, \widetilde{x}, \widetilde{t})+\cdots, \\
\widetilde{v}=\widetilde{\alpha}^{2 / 3} \widetilde{v}_{2}(r, \widetilde{x}, \widetilde{t})+\cdots+\widetilde{\alpha}^{1 / 6} \widetilde{v}_{5}(r, \widetilde{x}, \widetilde{t})+\cdots, \\
\widetilde{p}=\widetilde{\alpha}^{-1 / 3} \widetilde{p}_{2}(\widetilde{x}, \widetilde{t})+\cdots+\widetilde{\alpha}^{-5 / 6} \widetilde{p}_{5}(\widetilde{x}, \widetilde{t})+\cdots,
\end{array}
$$

across the majority of the pipe. The subscript $M$ denotes a mean flow distortion, while terms with subscript 5 anticipate the occurrence of the induced $O\left(\widetilde{\alpha}^{-1 / 2}\right)$ phase shift. The fundamental disturbances $\left(\widetilde{u}_{2}, \widetilde{v}_{2}, \widetilde{p}_{2}\right)$ take the form of the real parts of

$$
A_{0}(\widetilde{t})\left(F_{2}(r), \mathrm{i} G_{2}(r), 1\right) \exp (\widetilde{\mathrm{i}} \widetilde{\xi})
$$

respectively. Substitution of the expansions (4.3), (4.4) into the governing equations (4.1) leads to the balances

$$
F_{2}+G_{2}^{\prime}+\frac{G_{2}}{r}=0, \quad\left(u_{0}-c_{0}\right) F_{2}+G_{2} u_{0}^{\prime}=-1,
$$

where the prime denotes differentiation with respect to $r$. It is evident from (4.5) that the velocity components are singular at the radial location $r=r_{c}(\widetilde{t})$ say, where $u_{0}=c_{0}$. Solving for $G_{2}$ and applying the conditions $G_{2}(0)=G_{2}(1)=0$ we obtain

$$
\frac{r G_{2}}{\left(u_{0}-c_{0}\right)}=\left\{\begin{array}{ll}
\int_{0}^{r} \frac{s}{\left(u_{0}-c_{0}\right)^{2}} \mathrm{~d} s & \left(r<r_{c}\right) \\
\int_{1}^{r} \frac{s}{\left(u_{0}-c_{0}\right)^{2}} \mathrm{~d} s & \left(r>r_{c}\right)
\end{array} .\right.
$$

The limiting forms of $F_{2}$ and $G_{2}$ as the critical location is approached are also of interest. We calculate from (4.5) that as $r \rightarrow r_{c}-$ :

$$
F_{2} \sim\left(\frac{2 \tau_{1}+\tau_{0}}{\tau_{0}^{2}}\right) \ln \left(r_{c}-r\right), \quad G_{2} \sim \frac{r_{c}}{\tau_{0}}+\left(\frac{2 \tau_{1}+\tau_{0}}{\tau_{0}^{2}}\right)\left(r_{c}-r\right) \ln \left(r_{c}-r\right),
$$

where the skin-friction and curvature coefficients $\tau_{0}, \tau_{1}$ are defined by

$$
\tau_{0}=-r_{c} u_{0}^{\prime}\left(r_{c}\right), \tau_{1}=\frac{1}{2} r_{c}^{2} u_{0}^{\prime \prime}\left(r_{c}\right)
$$

A jump condition is required across the critical layer arising from the phase shift $\widetilde{\alpha}^{-1 / 2} \Phi$ where $\Phi$ is an $O(1)$ quantity to be determined. The implication of this is that terms such as $\ln \left(r_{c}-r\right) \cos \widetilde{\xi}$ are replaced by $\ln \left(r-r_{c}\right) \cos \widetilde{\xi}-\widetilde{\alpha}^{-1 / 2} \Phi \sin \widetilde{\xi}$ as we cross the critical layer. Consequently, the terms $\left(\widetilde{u}_{5}, \widetilde{v}_{5}, \widetilde{p}_{5}\right)$ in expansions (4.3) will be the first to undergo a non-zero phase shift, and therefore contain contributions of the form

$$
A_{0}\left(F_{5}(r) \sin \widetilde{\xi}, G_{5}(r) \cos \widetilde{\xi}, Q_{5} \sin \widetilde{\xi}\right)
$$

We will use the notation []$_{-}^{+}$to denote the jump in a quantity across the critical layer, i.e.

$$
[]_{-}^{+} \equiv \lim _{r \rightarrow r_{c}+}()-\lim _{r \rightarrow r_{c}-}()
$$


and use FP to denote the finite part of that jump. The limiting forms (4.7), together with the $\widetilde{\alpha}^{-1 / 2} \Phi$ phase shift, imply that

$$
\mathrm{FP}\left[\frac{G_{2}(r)}{r_{c}-r}\right]_{-}^{+}=0, \quad\left[\frac{G_{5}(r)}{r_{c}-r}\right]_{-}^{+}=-\left(\frac{2 \tau_{1}+\tau_{0}}{\tau_{0}^{2}}\right) \Phi
$$

Applying the first of these conditions to the solution (4.6) we obtain the integral criterion

$$
\mathrm{FP} \int_{0}^{1} \frac{r \mathrm{~d} r}{\left(u_{0}-c_{0}\right)^{2}}=0
$$

which determines the leading-order wavespeed. This result, in planar form, also arises in Smith's (1988) study of finite-time breakdown in unsteady boundary layers. From substitution into (4.1) and elimination of $F_{5}$ we see that the normal velocity component $G_{5}$ satisfies

$$
G_{5}^{\prime}+\left(\frac{1}{r}-\frac{u_{0}^{\prime}}{u_{0}-c_{0}}\right) G_{5}=\frac{Q_{5}}{\left(u_{0}-c_{0}\right)},
$$

with boundary conditions

$$
G_{5}(0)=0, G_{5}(1)=g_{s}
$$

The second of these conditions represents a displacement effect due to the viscous wall layer near $r=1$ and the precise value of $g_{s}$ will be calculated in (4.14) below. Solving the equation for $G_{5}$ and applying the integral condition (4.11) we deduce that

$$
\left[\frac{G_{5}(r)}{r_{c}-r}\right]_{-}^{+}=-\frac{\tau_{0} g_{s}}{r_{c}^{2} c_{0}}
$$

Comparing the second of equations (4.10) with (4.12), it is evident that the phase shift satisfies

$$
\Phi=\frac{\tau_{0}^{3} g_{s}}{c_{0} r_{c}^{2}\left(2 \tau_{1}+\tau_{0}\right)} .
$$

We now examine the dynamics of the viscous wall layer in order to determine the quantity $g_{s}$.

\subsection{The viscous Wall Layer}

As in the linear upper-branch structure, a viscous wall layer is necessary in order that the disturbances satisfy the no-slip condition. In this layer we have $r=1-\widetilde{\alpha}^{-1 / 2} Z$ with $Z$ of $O(1)$, and the appropriate forms for the velocity and pressure are

$$
\begin{aligned}
& \widetilde{u}=-\widetilde{\alpha}^{-1 / 2} u_{0}^{\prime}(1) Z+\cdots+\widetilde{\alpha}^{-1 / 3} A_{0} \Re(\bar{u}(Z) \exp (\mathrm{i} \widetilde{\xi}))+\cdots, \\
& \widetilde{v}=-\widetilde{\alpha}^{1 / 6} A_{0} \Re(\mathrm{i} \bar{v}(Z) \exp (\mathrm{i} \tilde{\xi}))+\cdots, \widetilde{p}=\widetilde{\alpha}^{-1 / 3} A_{0} \cos \widetilde{\xi}+\cdots .
\end{aligned}
$$

Substitution of these expansions into the governing equations (4.1) leads to the viscous balances

$$
\bar{u}+\bar{v}^{\prime}=0,-i c_{0} \bar{u}=-\mathrm{i}+\bar{u}^{\prime \prime}
$$

The appropriate solutions, satisfying no-slip on $Z=0$, are:

$$
\bar{u}=\frac{1}{c_{0}}\left(1-\exp \left(-m_{1} Z\right)\right), \bar{v}=\frac{1}{c_{0}}\left(\frac{1}{m_{1}}-\frac{1}{m_{1}} \exp \left(-m_{1} Z\right)-Z\right), m_{1} \equiv c_{0}^{1 / 2} \exp (-\mathrm{i} \pi / 4) .
$$


From the behaviour of the normal velocity component as $Z \rightarrow \infty$, and the required matching with the inviscid solution (4.3) above, we deduce that

$$
g_{s}=\frac{1}{\sqrt{2} c_{0}^{3 / 2}} .
$$

Thus we now have an expression for the phase shift $\Phi$, namely (4.13), arising from the wall-layer properties. Our next aim is to find an amplitude-phase shift relation from an investigation of the nonlinear critical layer.

\subsection{The nonlinear critical layer}

The structure here and many of the scalings appear similar to the Smith \& Bodonyi (1982) structure for Hagen-Poiseuille flow. Once again however, differences in the detail arise, firstly from the fact that the basic flow is more complicated here, and secondly that the disturbances are axisymmetric, which tends to simplify the analysis in places. We write

$$
r=r_{c}+\widetilde{\alpha}^{-1 / 6} Y,
$$

with $Y$ of $O(1)$, and expand the velocities and pressure as follows:

$$
\begin{aligned}
& \widetilde{u}=c_{0}+\widetilde{\alpha}^{-1 / 6} U_{1}(Y, \widetilde{\xi})+\cdots+\widetilde{\alpha}^{-1 / 3} U_{2}(Y, \widetilde{\xi})+\cdots+\widetilde{\alpha}^{-5 / 6} U_{5}(Y, \widetilde{\xi})+\cdots, \\
& \widetilde{v}=\widetilde{\alpha}^{2 / 3} V_{1}(Y, \widetilde{\xi})+\cdots+\widetilde{\alpha}^{1 / 2} V_{2}(Y, \widetilde{\xi})+\cdots+V_{5}(Y, \widetilde{\xi})+\cdots, \\
& \widetilde{p}=\widetilde{\alpha}^{-1 / 3} P_{1}(\widetilde{\xi})+\cdots+\widetilde{\alpha}^{-1 / 2} P_{2}(\widetilde{\xi})+\cdots+\widetilde{\alpha}^{-1} P_{5}(\widetilde{\xi})+\cdots
\end{aligned}
$$

Again, terms with subscript 5 turn out to be those affected by the phase shift. The leading order nonlinear balances are:

$$
U_{1 \widetilde{\xi}}+V_{1 Y}=0, \quad U_{1} U_{1 \widetilde{\xi}}+V_{1} U_{1 Y}=-P_{1 \tilde{\xi}},
$$

with the main pressure disturbance assuming the value

$$
P_{1}=A_{0} \cos \widetilde{\xi},
$$

from (4.3), (4.4). To match to the core flow we require

$$
U_{1} \sim-\frac{\tau_{0}}{r_{c}} Y+\widetilde{u}_{1 M}\left(r_{c}, \widetilde{t}\right), \quad V_{1} \sim-\widetilde{\beta} \sin \widetilde{\xi},
$$

as $Y \rightarrow \pm \infty$, with amplitude parameter

$$
\widetilde{\beta} \equiv \frac{A_{0} r_{c}}{\tau_{0}} .
$$

In fact the asymptotic forms (4.17) given above can be shown to be the exact solutions for $U_{1}, V_{1}$ throughout the critical layer (as in Benney \& Bergeron 1969) and this simplification allows the higher-order contributions and in particular the phase shift to be determined with minimal computation. A phase shift at level $m$ is only possible if the solution for $\left(U_{m}, P_{m}\right)$ possesses an odd part about $\widetilde{\xi}=\pi$, with $V_{m}$ having an even part. The task here is to find the smallest value of $m$ for which this holds. At the $m=2$ stage the equations governing $U_{2}, V_{2}$ are:

$$
\begin{gathered}
U_{2 \widetilde{\xi}}+V_{2 Y}=\frac{A_{0} \sin \widetilde{\xi}}{\tau_{0}}, \\
\left(-\frac{\tau_{0}}{r_{c}} Y+u_{1 M}\left(r_{c}, \widetilde{t}\right)\right) U_{2 \widetilde{\xi}}-\widetilde{\beta} \sin \widetilde{\xi} U_{2 Y}-\frac{\tau_{0}}{r_{c}} V_{2}=-P_{2 \widetilde{\xi}} .
\end{gathered}
$$


We differentiate $(4.19 b)$ with respect to $Y$ to eliminate $P_{2}$ and make the change of variable $(\widetilde{\xi}, Y) \rightarrow(\widehat{\xi}, \widetilde{\eta})$ with

$$
\widetilde{\xi}=\widehat{\xi}, \quad \widetilde{\eta}=\frac{\tau_{0}}{2 r_{c}}\left(Y-\frac{r_{c} \widetilde{u}_{1 M}\left(r_{c}, \widetilde{t}\right)}{\tau_{0}}\right)^{2}+\widetilde{\beta} \cos \widetilde{\xi} .
$$

In terms of this new notation, and after elimination of $V_{2}$ we have

$$
\mp\left(\frac{2 \tau_{0}}{r_{c}}\right)^{1 / 2}(\widetilde{\eta}-\widetilde{\beta} \cos \widehat{\xi})^{1 / 2} \frac{\partial}{\partial \widehat{\xi}}\left(U_{2 Y}\right)=\frac{A_{0} \sin \widehat{\xi}}{r_{c}}
$$

and upon integration we obtain

$$
-2 r_{c}^{1 / 2} U_{2 Y}=\kappa(\widetilde{\eta}) \pm(\widetilde{\eta}-\widetilde{\beta} \cos \widehat{\xi})^{1 / 2} \frac{2 \sqrt{2}}{r_{c}} \tau_{0}^{1 / 2},
$$

with $\kappa$ an arbitrary function of $\widetilde{\eta}$. This equation is subject to the matching condition

$$
U_{2} \sim \frac{\tau_{1}}{r_{c}^{2}} Y^{2}+Y \widetilde{u}_{1 M}^{\prime}\left(r_{c} \pm, \widetilde{t}\right)+\widetilde{u}_{2 M}\left(r_{c} \pm, \widetilde{t}\right)+A_{0}\left(\frac{2 \tau_{1}+\tau_{0}}{\tau_{0}^{2}}\right) \ln |Y| \cos \widetilde{\xi},
$$

as $Y \rightarrow \pm \infty$. In other words, the vorticity satisfies

$$
U_{2 Y} \sim \frac{2 \tau_{1}}{r_{c}^{2}} Y+\tilde{\lambda}^{ \pm} \text {as } Y \rightarrow \pm \infty
$$

with $\widetilde{\lambda}^{ \pm}=\widetilde{u}_{1 M}^{\prime}\left(r_{c} \pm, \widetilde{t}\right)$, and $\widetilde{\lambda}^{+}-\widetilde{\lambda}^{-}$representing the vorticity jump across the critical layer. In view of this asymptotic condition we find that a requirement of the shear term $\kappa(\widetilde{\eta})$ introduced in $(4.20)$ is

$$
\kappa(\widetilde{\eta}) \sim \mp \frac{2^{3 / 2}}{r_{c} \tau_{0}^{1 / 2}}\left(2 \tau_{1}+\tau_{0}\right) \widetilde{\eta}^{1 / 2} \text { as } \widetilde{\eta} \rightarrow \infty .
$$

In contrast to previous studies there is no viscous forcing at the $m=4$ stage because $U_{1 Y Y} \equiv 0$ and so we proceed directly to the $m=5$ level. The governing equation at this order can be written in the form

$$
\mp\left(\frac{2 \tau_{0}}{r_{c}}\right)^{1 / 2}(\widetilde{\eta}-\widetilde{\beta} \cos \widehat{\xi})^{1 / 2} \frac{\partial U_{5 Y}}{\partial \widehat{\xi}}=U_{2 Y Y Y}+{ }^{\prime} O ',
$$

using ' $O$ ' to represent contributions that are odd about $\widehat{\xi}=\pi$. Substituting for $U_{2 Y Y Y}$ using (4.20) and integrating from 0 to $2 \pi$ in $\widehat{\xi}$ we obtain the result

$$
\frac{\partial}{\partial \widetilde{\eta}}\left(\kappa^{\prime}(\widetilde{\eta}) I(\widetilde{\eta})\right)=0, \quad I(\widetilde{\eta}) \equiv \int_{0}^{2 \pi}(\widetilde{\eta}-\widetilde{\beta} \cos q)^{1 / 2} \mathrm{~d} q
$$

and hence

$$
\kappa^{\prime}(\widetilde{\eta})=\widetilde{D}^{ \pm} / I(\widetilde{\eta}), \quad(\widetilde{\eta}>\widetilde{\beta})
$$

The constants $\widetilde{D}^{ \pm}$can be determined from the asymptotic condition (4.22) and are given by

$$
\widetilde{D}^{ \pm}=\mp \frac{2^{3 / 2} \pi\left(\tau_{0}+2 \tau_{1}\right)}{r_{c} \tau_{0}^{1 / 2}} .
$$

The streamlines here are of cat's eye form and are closed for $\widetilde{\eta}<\widetilde{\beta}$. Interior to the eye, the constant $\widetilde{D} \equiv 0$ and hence from (4.24) the region $\widetilde{\eta}<\widetilde{\beta}$ possesses uniform vorticity, a 
consequence of the Prandtl-Batchelor theorem (Batchelor 1956). Although in the present structure this region is passive, it assumes much greater significance later in section 5 .

Turning now to the calculation of the phase shift, we first consider the quantity $\widetilde{\phi}$ defined by

$$
\widetilde{\phi} \equiv \frac{1}{\pi} \int_{0}^{2 \pi}\left(\mathrm{FP}\left[U_{5}\right]_{-}^{+} \sin \widetilde{\xi} \mathrm{d} \widetilde{\xi}\right) \mathrm{d} Y=\frac{1}{\pi} \mathrm{FP} \int_{-\infty}^{+\infty}\left(\int_{0}^{2 \pi} U_{5 Y} \sin \widetilde{\xi} \mathrm{d} \tilde{\xi}\right) \mathrm{d} Y .
$$

Changing to $(\widehat{\xi}, \widetilde{\eta})$ variables, integrating by parts and substituting for $\partial U_{5 Y} / \partial \widehat{\xi}$ from (4.23), the above expression simplifies to

$$
\widetilde{\phi}=\frac{r_{c}}{\pi \widetilde{\beta} \tau_{0}} \mathrm{FP} \int_{-\infty}^{+\infty} \int_{0}^{2 \pi} U_{2 Y Y Y} \mathrm{~d} \widehat{\xi} \mathrm{d} \widetilde{\eta} .
$$

Next, reverting to $(\widetilde{\xi}, Y)$ variables and integrating by parts we obtain the result

$$
\pi \widetilde{\beta} \tilde{\phi}=-\int_{0}^{2 \pi} \operatorname{FP}\left[U_{2 Y}\right]_{-}^{+} \mathrm{d} \widetilde{\xi}=-2 \pi\left(\widetilde{\lambda}^{+}-\widetilde{\lambda}^{-}\right)
$$

with the last expression arising from use of (4.21). In order to relate the quantity $\widetilde{\phi}$ to the amplitude of the neutral modes we note that from (4.20) and (4.21):

$$
\mathrm{FP}\left[U_{2 Y}\right]_{-}^{+}=\left(\widetilde{\lambda}^{+}-\widetilde{\lambda}^{-}\right)=-\frac{1}{2 r_{c}^{1 / 2}} \mathrm{FP}[\kappa]_{-}^{+},
$$

and hence we have that

$$
\widetilde{\phi}=\frac{1}{r_{c}^{1 / 2} \widetilde{\beta}} \mathrm{FP}[\kappa]_{-}^{+} .
$$

The finite part of the jump in $\kappa$ can be calculated from (4.24) and is given by

$$
\mathrm{FP}[\kappa]_{-}^{+}=\left(\widetilde{D}^{+}-\widetilde{D}^{-}\right) \mathrm{FP} \int_{\widetilde{\beta}}^{\infty} \frac{\mathrm{d} s}{I(s)}=-\widetilde{\beta}^{1 / 2} C^{(1)} \frac{\left(\tau_{0}+2 \tau_{1}\right)}{r_{c} \tau_{0}^{1 / 2}},
$$

where (4.25) has been used for $\widetilde{D}^{+/-}$and $C^{(1)}$ is the same constant that arises in (3.6). Substituting for $\widetilde{\beta}$ from (4.18), and using (4.26) we find that $\widetilde{\phi}$ is related to $A_{0}$ via

$$
\widetilde{\phi}=-\frac{C^{(1)}\left(\tau_{0}+2 \tau_{1}\right)}{A_{0}^{1 / 2} r_{c}^{2}} .
$$

\subsection{Determination of the neutral wave amplitude}

It remains to relate $\widetilde{\phi}$ to the scaled phase shift $\Phi$ by considering the logarithmic behaviour within the inviscid region. From the asymptotic form for $F_{2}$ in (4.7) and the expansion (4.3a) we deduce that the jump in the component $F_{5}$ (defined in (4.9)) is given by

$$
\left[F_{5}\right]_{-}^{+}=-\Phi\left(\frac{\tau_{0}+2 \tau_{1}}{\tau_{0}^{2}}\right)
$$

using the phase shift condition given just above (4.9). The relationship between $\Phi$ and $\widetilde{\phi}$ is therefore

$$
\widetilde{\phi}=-\Phi\left(\frac{\tau_{0}+2 \tau_{1}}{\tau_{0}^{2}}\right) A_{0}
$$

and hence the required phase shift-amplitude relation is

$$
\Phi=\left(\frac{\tau_{0}}{r_{c}}\right)^{2} C^{(1)} A_{0}^{-3 / 2}
$$



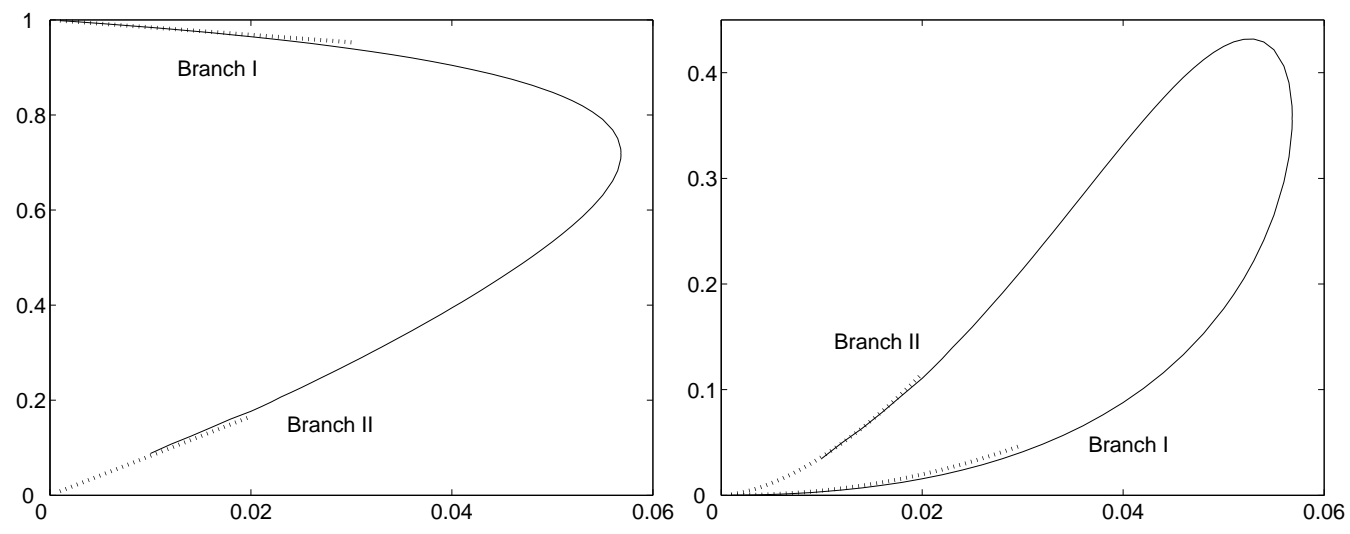

FiguRE 2. Nonlinear neutral curves for developing pipe flow with $\alpha R \gg 1, \tilde{t} \sim O(1)$. The dotted lines are the small $\widetilde{t}$-asymptotes. (a) Critical layer location $r_{c}$ versus $\widetilde{t}$; (b) neutral wave amplitude $A_{0}$ versus $\widetilde{t}$.

Putting this together with the wall-layer expression for $\Phi$ derived earlier in (4.13), (4.14), we deduce that the amplitude of the neutral modes is given by

$$
A_{0}=2^{1 / 3} c_{0}^{5 / 3}\left|\frac{\tau_{0}+2 \tau_{1}}{\tau_{0}}\right|^{2 / 3}\left|C^{(1)}\right|^{2 / 3} .
$$

In summary, for given $\widetilde{t}$, the basic flow $u_{0}$ can be calculated from (1.3). The appropriate wavespeed $c_{0}$ then follows from the requirement (4.11), and is calculated using the method outlined in the appendix. The critical layer location $r_{c}$ can then be deduced from $c_{0}=$ $u_{0}\left(r_{c}\right)$. The corresponding amplitude of the neutral modes follows from (4.28), with $\tau_{0}, \tau_{1}$ calculated using (4.8). In figure 2 we show how the critical layer location $r_{c}$ and amplitude $A_{0}$ vary with $\widetilde{t}$. There are a number of interesting features to these curves. The first is that they close up at a finite value of $\widetilde{t}, \widetilde{t}_{c}$ say, with $\widetilde{t}_{c} \simeq 0.057$. This indicates that this type of axisymmetric instability does not occur once the base flow is sufficiently developed. In particular it means that Hagen-Poiseuille flow is not susceptible to such an instability and this is consistent with Smith \& Bodonyi's analysis where they found only nonsymmetric modes (with an azimuthal wavenumber of unity). A second feature of figure 2 are the two branches along which $\widetilde{t} \rightarrow 0$, and these prove worthy of more detailed investigation.

\subsection{Small-time analysis of the nonlinear neutral curve}

(i) Branch I. This is the upper branch of the curve in figure 2(a) and the lower branch in figure 2(b). The behaviour here is such that $c_{0}$ and $A_{0}$ tend to zero as $\widetilde{t} \rightarrow 0$. The critical layer is therefore approaching the wall in this limit and the mode structure appears to be reverting to its linear form. A match with the linear regime discussed earlier can be established by analyzing the integral criterion

$$
\mathcal{I} \equiv \mathrm{FP} \int_{0}^{1} \frac{r \mathrm{~d} r}{\left(u_{0}-c_{0}\right)^{2}}=0
$$

as $\tilde{t} \rightarrow 0$. In this limit the basic flow acquires its two-tiered form (1.4) and the integral $I$ can be split into $\mathcal{I} \equiv \mathcal{I}_{1}+\mathcal{I}_{2}$ say, where $\mathcal{I}_{1}$ represents the contribution across the majority of the pipe with $u_{0} \sim 4 \widetilde{t}, c_{0} \sim 0$, while $I_{2}$ is the boundary-layer contribution from the region of order $\widetilde{t}^{1 / 2}$ in which $u_{0} \sim 4 \widetilde{t} u_{B}(\eta), c_{0} \sim 4 \widetilde{t}_{\bar{c}_{0}}$, with $u_{B}(\eta)$ given in (1.5). We 
therefore have

$$
\mathcal{I}_{1} \sim(4 \widetilde{t})^{-2} \int_{0}^{1} r \mathrm{~d} r=(4 \widetilde{t})^{-2} \frac{1}{2}
$$

and

$$
\mathcal{I}_{2} \sim(4 \widetilde{t})^{-2} \widetilde{t}^{1 / 2} \mathrm{FP} \int_{0}^{\infty} \frac{\mathrm{d} \eta}{\left(u_{B}(\eta)-\bar{c}_{0}\right)^{2}} .
$$

The dominant contribution to $I_{2}$ arises from a sublayer of the boundary-layer in which $\eta=\widetilde{t}^{1 / 2} Y$ (essentially region III of the linear structure), $u_{B} \sim \lambda_{0} \widetilde{t}^{1 / 2} Y$ and $\bar{c}_{0} \sim K \widetilde{t}^{1 / 2}$ with $K$ to be determined and $\lambda_{0}$ given in (2.2). To leading order therefore:

$$
\mathcal{I}_{2} \sim(4 \widetilde{t})^{-2} \mathrm{FP} \int_{0}^{\infty} \frac{d Y}{\left(\lambda_{0} Y-K\right)^{2}}=-(4 \widetilde{t})^{-2} \frac{1}{\lambda_{0} K} .
$$

Thus, in order for $\mathcal{I}$ to be zero we require, from (4.30) and (4.31) that $1 / 2-1 /\left(\lambda_{0} K\right)=0$ and hence $K$ is fixed as

$$
K=2 / \lambda_{0} .
$$

It follows that $\bar{c}_{0} \sim\left(2 / \lambda_{0}\right) \widetilde{t}^{1 / 2}$ as $\widetilde{t} \rightarrow 0$, and thus

$$
c_{0} \sim \frac{8}{\lambda_{0}} \widetilde{t}^{3 / 2}, r_{c} \sim 1-\frac{2}{\lambda_{0}^{2}} \widetilde{t} \text { as } \widetilde{t} \rightarrow 0,
$$

with the first of these precisely the linear asymptote found in (3.8). In addition the limiting form of the amplitude calculated from (4.28) with $r_{c} \rightarrow 0$, is found to be

$$
A_{0} \sim \frac{2^{6}\left(\lambda_{1} C^{(1)}\right)^{2 / 3}}{\lambda_{0}^{7 / 3}} \widetilde{t}^{13 / 6} \text { as } \widetilde{t} \rightarrow 0,
$$

and this matches exactly with the corresponding weakly nonlinear result in (3.8) established earlier, in view of the pressure scaling in $(4.3 c)$. Thus we conclude that branch I of the nonlinear solution matches back to the weakly nonlinear upper branch mode as $\widetilde{t}$ is decreased. The branch I asymptotes for $r_{c}$ and $A_{0}$ are shown as dotted lines on figures $2(a, b)$.

(ii) Branch II. A more unexpected feature of the curves in figure 2 is the presence of the second (upper) branch as $\widetilde{t} \rightarrow 0$, which we will refer to as branch II. This branch has $A_{0} \rightarrow 0$ as $\widetilde{t} \rightarrow 0$, but in this case it appears that the critical layer is approaching the pipe axis, rather than the wall (figure 2a). In order to obtain the limiting form for the wavespeed from the integral criterion (4.29) it is necessary to analyze the basic flow as $\widetilde{t} \rightarrow 0$ in more detail. Asymptotic analysis of (1.3) identifies the existence of an additional thin centre region of thickness $O(\widetilde{t})$ to supplement the core and boundary-layer regions identified earlier. It is also necessary to calculate the correction to the uniform core flow. The full three-tiered solution we require is

$$
u_{0} / 4 \widetilde{t} \sim\left\{\begin{array}{cc}
1-8 \widetilde{t} I_{0}\left(\frac{1}{2} z\right) \exp (-1 / 4 \widetilde{t}) & r=\widetilde{t} z \\
1-8 \pi^{-1 / 2} \widetilde{t}^{3 / 2} r^{-1 / 2}(1-r)^{-3} \exp \left(-(1-r)^{2} / 4 \widetilde{t}\right) & r \text { of } O(1) \\
u_{B}(\eta) & r=1-\widetilde{t}^{1 / 2} \eta,
\end{array}\right.
$$

with $u_{B}$ as given previously in (1.5). On branch II the dominant contribution to the integral as $\widetilde{t} \rightarrow 0$ arises from the thin centre region. Using the near-axis form for $u_{0}$ given above in (4.33) we find

$$
\mathcal{I} \sim(32 \widetilde{t})^{-2} \exp (1 / 2 \widetilde{t}) \mathrm{FP} \int_{0}^{\infty} \frac{z \mathrm{~d} z}{\left(I_{0}\left(\frac{1}{2} z\right)-\bar{c}_{1}\right)^{2}},
$$


where $c_{0}=4 \widetilde{t}\left(1-8 \widetilde{t} \exp (-1 / 4 \widetilde{t}) \bar{c}_{1}+\cdots\right)$. The value of $\bar{c}_{1}$ can therefore be calculated from the requirement

$$
\mathrm{FP} \int_{0}^{\infty} \frac{z \mathrm{~d} z}{\left(I_{0}\left(\frac{1}{2} z\right)-\bar{c}_{1}\right)^{2}}=0
$$

A numerical calculation (with details of the solution procedure given in the appendix) shows that

$$
z_{c} \simeq 8.281, \quad \bar{c}_{1}=I_{0}\left(\frac{1}{2} z_{c}\right) \simeq 12.77
$$

and hence we obtain the asymptotic results

$$
c_{0} \sim 4 \widetilde{t}-32 \widetilde{t}^{2} \exp (-1 / 4 \widetilde{t}) \bar{c}_{1}, \quad r_{c} \sim z_{c} \widetilde{t} \text { as } \widetilde{t} \rightarrow 0 .
$$

Using these expressions we can deduce the corresponding limiting forms for $\tau_{0}$ and $\tau_{1}$ and hence from (4.28) the mode amplitude tends to zero in the form

$$
A_{0} \sim 2^{11 / 3}\left(\Theta C^{(1)}\right)^{2 / 3} \widetilde{t}^{5 / 3} \text { as } \widetilde{t} \rightarrow 0,
$$

with

$$
\Theta \equiv 1+\frac{2 \tau_{1}\left(r_{c}\right)}{\tau_{0}\left(r_{c}\right)} \simeq-2.766
$$

The branch II asymptotes (4.36), (4.37) are shown as dotted lines on figures 2(a,b). The results are useful because it becomes difficult numerically to compute solutions on branch II for small values of $\widetilde{t}$. This is because the integrand of (4.29) becomes exponentially large in this limit, as is clear from (4.34). Evidently, by comparison with the branch I result (4.32), the amplitude decay is slower on branch II as $\widetilde{t} \rightarrow 0$. Eventually, for small enough $\widetilde{t}$, if $\widetilde{\alpha}$ is sufficiently large, this limiting structure in which the critical layer approaches the pipe axis as $\tilde{t}$ decreases, becomes invalid. Effectively the critical layer eventually includes $r=0$ and the governing equations have to be reformulated. In order to see how this can be achieved it is necessary to examine how each of the flow regions identified above behaves on branch II as $\widetilde{t}$ is decreased. We would expect the leading order behaviour to be consistent with the integral criterion (4.35) derived above.

\subsection{The behaviour on branch II in more detail}

First, from (4.8) and the near-axis form in (4.33) appropriate for the basic flow we deduce that

$$
\tau_{0} \sim-\frac{1}{2} \widetilde{t}^{2} \exp (-1 / 4 \widetilde{t}) z_{c} I_{0}^{\prime}\left(\frac{1}{2} z_{c}\right), \quad \tau_{1} \sim \frac{1}{8} \widetilde{t}^{2} \exp (-1 / 4 \widetilde{t}) z_{c}^{2} I_{0}^{\prime \prime}\left(\frac{1}{2} z_{c}\right) \text { as } \widetilde{t} \rightarrow 0
$$

Then using this result, we find that the inviscid region I, formerly of $O(1)$ thickness, contracts to $O(\widetilde{t})$ in this limit, and in this layer (which we will refer to as the slug region, for reasons to be explained later) we have

$$
\begin{aligned}
& u_{0}=4 \widetilde{t}-32 \widetilde{t}^{2} \mathrm{e}^{-1 / 4 \widetilde{t}} I_{0}\left(\frac{1}{2} z\right)+\cdots, \\
& \widetilde{u}=u_{0}+\widetilde{\alpha}^{-1 / 3} \widetilde{t}^{-1 / 3} \mathrm{e}^{1 / 4 \widetilde{t}} \mathfrak{u}_{2}(z) \cos \widetilde{\xi}+\cdots \\
& +\widetilde{\alpha}^{-5 / 6} \widetilde{t}^{-5 / 6} \mathrm{e}^{-1 / 4 \widetilde{t}_{u_{5 S}}(z) \sin \widetilde{\xi}+\cdots,}
\end{aligned}
$$

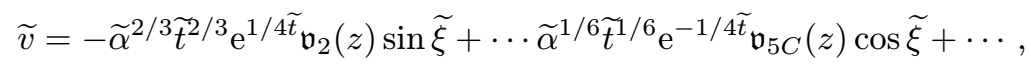

$$
\begin{aligned}
& \widetilde{p}=\widetilde{\alpha}^{-1 / 3} \widetilde{t}^{5 / 3} Q_{2} \cos \widetilde{\xi}+\cdots \widetilde{\alpha}^{-5 / 6} \widetilde{t}^{7 / 6} \mathrm{e}^{-1 / 2 \widetilde{t}} Q_{5 S} \sin \widetilde{\xi}+\cdots,
\end{aligned}
$$

with the latter terms in the expansions for $(\widetilde{u}, \widetilde{v}, \widetilde{p})$ arising from the phase shift across the critical layer. The wavespeed expansion is given in (4.36) while the scalings on $\widetilde{\alpha}$ 
remain as in (4.3). Substitution of these limiting forms into the governing equations and application of the condition of zero jump in $\mathfrak{v}_{2}$ across the critical layer leads to the integral criterion (4.35), as expected. In a similar way an equation for $\mathfrak{v}_{5 C}$ can be derived, and from the resulting solution we deduce that

$$
\mathfrak{v}_{5 C} \sim \frac{K_{1}}{\sqrt{\pi}} z^{-3 / 2} \exp (z / 2) \text { as } z \rightarrow \infty,
$$

where $K_{1}$ is an, as yet, unknown constant. In addition the jump across the critical layer in the normal derivative of the radial velocity is found to be

$$
\left[\frac{\partial \widetilde{v}}{\partial z}\right]_{-}^{+}=\widetilde{\alpha}^{1 / 6} \widetilde{t}^{1 / 6} \exp (-1 / 4 \widetilde{t}) \frac{I_{0}^{\prime}\left(\frac{1}{2} z_{c}\right)}{2 z_{c}} K_{1} \cos \widetilde{\xi} .
$$

Next we analyze the viscous wall layer in the small-time limit. The working is very similar to that outlined in section 4.2 with the addition of the extra scaling in $\widetilde{t}$. We find, to leading order:

$$
\begin{array}{r}
r=1-\widetilde{\alpha}^{-1 / 2} \widetilde{t}^{-1 / 2} Z, \quad u_{0}=4 \widetilde{\alpha}^{-1 / 2} u_{B}^{\prime}(0) Z, \\
\widetilde{u}=u_{0}+\widetilde{\alpha}^{-1 / 3} \widetilde{t}^{2 / 3} \bar{u}(\widetilde{\xi}, Z), \quad \widetilde{v}=-\widetilde{\alpha}^{1 / 6} \bar{t}^{1 / 6} \bar{v}(\widetilde{\xi}, Z),
\end{array}
$$

with the expansion $(4.38 d)$ for $\widetilde{p}$. The solution satisfying no-slip at the wall gives, as $Z \rightarrow \infty$ :

$$
\bar{u} \rightarrow \frac{Q_{2}}{4} \cos \widetilde{\xi}, \bar{v} \sim \frac{Q_{2}}{4} Z \sin \widetilde{\xi}-\frac{Q_{2}}{2^{7 / 2}}(\cos \widetilde{\xi}+\sin \widetilde{\xi}) .
$$

This must match with the outer boundary-layer-type region (region II in the earlier linear analysis) in which the basic flow $u_{0}$ has the error function form given in the lower tier of (4.33). Here the appropriate expansions are

$$
\begin{aligned}
& \widetilde{u}=u_{0}+\widetilde{\alpha}^{-1 / 3} \widetilde{t}^{2 / 3} \widehat{u}(\eta) \cos \widetilde{\xi}+\cdots+\widetilde{\alpha}^{-5 / 6} \widetilde{t}^{-1 / 3} \widehat{u}_{c}(\eta) \sin \widetilde{\xi}+\cdots, \\
& \widetilde{v}=-\widetilde{\alpha}^{2 / 3} \widetilde{t}^{7 / 6} \widehat{v}(\eta) \sin \widetilde{\xi}+\cdots+\widetilde{\alpha}^{1 / 6} \widetilde{t}^{1 / 6} \widehat{v}_{c}(\eta) \cos \widetilde{\xi}+\cdots
\end{aligned}
$$

The solution for $\widehat{v}$ is

$$
\widehat{v}=-\frac{Q_{2}}{4}\left(u_{B}(\eta)-1\right) \int_{0}^{\eta} \frac{\mathrm{d} s}{\left(u_{B}(s)-1\right)^{2}},
$$

and from this solution, and the continuity equation, we deduce that

$$
\widehat{u} \sim \frac{Q_{2}}{64} \sqrt{\pi} \eta^{3} \exp \left(\eta^{2} / 4\right), \quad \widehat{v} \sim \frac{Q_{2}}{32} \sqrt{\pi} \eta^{2} \exp \left(\eta^{2} / 4\right)
$$

as $\eta \rightarrow \infty$. The equation for $\widehat{v}_{c}$ is unforced by the pressure and as a result the simple form

$$
\widehat{v}_{c}=\frac{Q_{2}}{2^{7 / 2}}\left(u_{B}(\eta)-1\right)
$$

is obtained, with the constant of proportionality chosen to match to the wall layer solution given in (4.41). The far-field forms for $\widehat{u}_{c}$ and $\widehat{v}_{c}$ can then be deduced as

$$
\widehat{u}_{c} \sim-\frac{Q_{2}}{2 \sqrt{2 \pi}} \frac{\exp \left(-\eta^{2} / 4\right)}{\eta^{2}}, \widehat{v}_{c} \sim-\frac{Q_{2}}{\sqrt{2 \pi}} \frac{\exp \left(-\eta^{2} / 4\right)}{\eta^{3}},
$$

as $\eta \rightarrow \infty$. Having established the behaviour in the outer reaches of both the slug region (4.39) and the boundary layer(4.42), it is a straightforward matter to deduce the flow solution throughout the core where the basic flow $u_{0}$ is given by the middle tier of (4.33). 

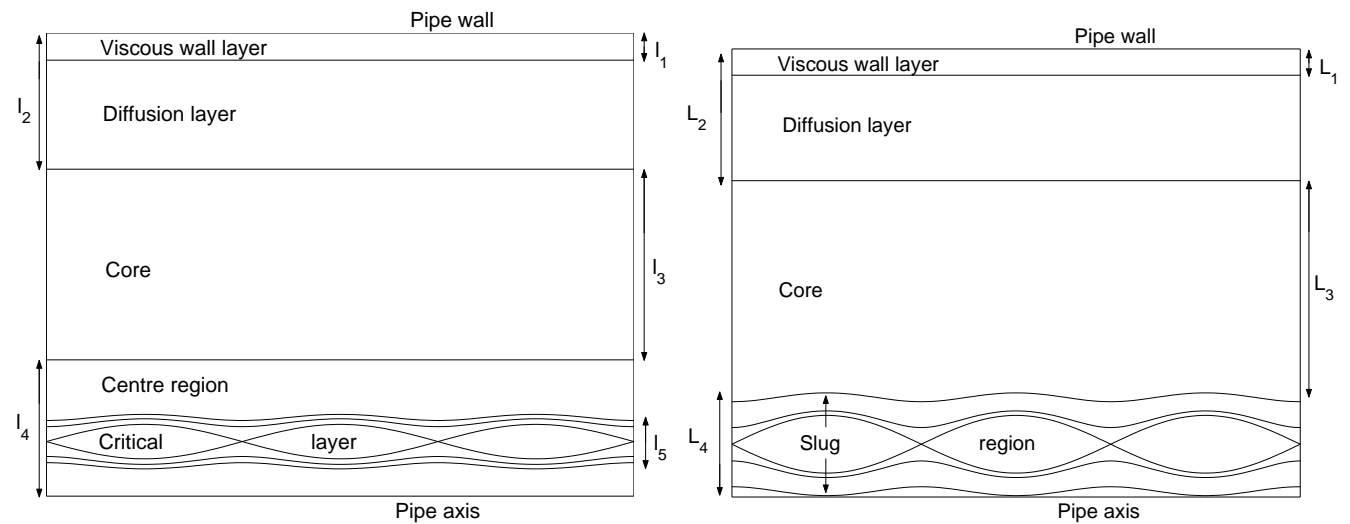

FiguRE 3. (a) The mode structure on branch II of the nonlinear neutral curve when $\widetilde{t} \ll 1$. In terms of $\widetilde{\alpha}(\equiv \alpha R)$ and $\widetilde{t}$, the thicknesses of the regions are $1_{1} \sim \widetilde{\alpha}^{-1 / 2} \widetilde{t}^{-1 / 2}, 1_{2} \sim \widetilde{t}^{1 / 2}, 1_{3} \sim O(1)$, $1_{4} \sim \widetilde{t}, 1_{5} \sim \widetilde{\alpha}^{-1 / 6} \widetilde{t}^{-1 / 6} \exp (1 / 4 \widetilde{t})$; (b) the new nonlinear structure that arises at small values of $\widetilde{t}$ on branch II when $\widetilde{\alpha} \sim \widetilde{t}^{-7} \exp (3 / 2 \widetilde{t}) \gg 1$. Here, the thicknesses of the regions are given in terms of $\widetilde{t}$ by $L_{1} \sim \widetilde{t}^{3} \exp (-3 / 4 \widetilde{t}), L_{2} \sim \widetilde{t}^{1 / 2}, L_{3} \sim O(1), L_{4} \sim \widetilde{t}$.

We find

$$
\begin{aligned}
\widetilde{u}= & u_{0}+\widetilde{\alpha}^{-1 / 3} \widetilde{t}^{-5 / 6} \frac{Q_{2} \sqrt{\pi r}}{64}(1-r)^{3} E_{1} \cos \widetilde{\xi}+\cdots \\
& -\widetilde{\alpha}^{-5 / 6} \widetilde{t}^{2 / 3} \frac{Q_{2}}{2 \sqrt{2 \pi}} r^{-3 / 2}(1-r)^{-2} E_{1}^{-1} \sin \widetilde{\xi}+\cdots, \\
\widetilde{v}= & -\widetilde{\alpha}^{2 / 3} \widetilde{t}^{1 / 6} \frac{Q_{2} \sqrt{\pi r}}{32}(1-r)^{2} E_{1} \sin \widetilde{\xi}+\cdots \\
& +\widetilde{\alpha}^{1 / 6} \widetilde{t}^{5 / 3} \frac{Q_{2}}{\sqrt{2 \pi}} r^{-3 / 2}(1-r)^{-3} E_{1}^{-1} \cos \widetilde{\xi}+\cdots,
\end{aligned}
$$

Here $E_{1} \equiv \exp \left[(1-r)^{2} / 4 \widetilde{t}\right]$, with $\tilde{p}$ remaining as in $(4.38 d)$. In carrying out the matching process it is found that the constant $K_{1}$ must take the value

$$
K_{1}=Q_{2} / \sqrt{2} \text {. }
$$

Finally, the critical layer analysis proceeds in a similar fashion to that described in section 4.3 and results in the determination of a phase shift-amplitude relation similar to (4.27). Equivalently, the phase shift can also be deduced from the jump in $\partial \widetilde{v} / \partial z$ given in (4.40) with $K_{1}$ known from (4.44). Equating the two expressions leads to the result

$$
Q_{2}^{3 / 2}=2^{11 / 2} \Theta C^{(1)},
$$

and hence we recover the small-time amplitude result (4.37), as expected. This completes the description of the flow behaviour on branch II of the curves in figure 2 as $\widetilde{t} \rightarrow 0$. The resulting asymptotic structure is sketched in figure 3(a).

\section{The nonlinear slug flow}

The small - $\tilde{t}$ branch II structure we have set out above becomes invalid for sufficiently small $\tilde{t}$ or equivalently, for large enough values of $\widetilde{\alpha}$. The new structure that replaces it is dominated by a nonlinear slug region which includes the pipe axis and replaces the nonlinear critical layer. To see how the existing structure fails, consider the axial velocity 
within the linear slug region. From $(4.38 \mathrm{a}, \mathrm{b})$ this has the form

$$
\widetilde{u}=4 \widetilde{t}-32 \widetilde{t}^{2} \exp (-1 / 4 \widetilde{t}) I_{0}\left(\frac{1}{2} z\right)+\cdots+\widetilde{\alpha}^{-1 / 3} \widetilde{t}^{-1 / 3} \exp (1 / 4 \widetilde{t}) \mathfrak{u}_{2} \cos \widetilde{\xi}+\cdots
$$

We observe that the second and third terms in the expansion become comparable when $\widetilde{\alpha} \sim O\left(\widetilde{t}^{-7} \exp (3 / 2 \widetilde{t})\right) \gg 1$. To investigate the new structure that takes over, it is convenient to introduce a small parameter $\varepsilon$ and a new time variable $s$ such that

$$
\exp (-1 / \widetilde{t})=\varepsilon s,
$$

with $\widetilde{t}=(-\ln \varepsilon)^{-1}$ to leading order. Then, in the new regime we have that $\widetilde{\alpha}$ is related to $\varepsilon$ via

$$
\widetilde{\alpha}=\varepsilon^{-3 / 2}(-\ln \varepsilon)^{7} .
$$

In terms of $\varepsilon$, the axial and radial scalings in the new slug region are

$$
\frac{\partial}{\partial x}=\varepsilon^{-3 / 2}(-\ln \varepsilon)^{7} \frac{\partial}{\partial \widetilde{\xi}}, \frac{\partial}{\partial r}=(-\ln \varepsilon+\cdots) \frac{\partial}{\partial z} .
$$

The leading order wavespeed is unchanged, but the correction, previously determined from the integral criterion (4.35) is now unknown and dependent on the full solution of the slug problem. We therefore write

$$
c=\frac{4}{(-\ln \varepsilon)}+\cdots+\frac{\varepsilon^{1 / 4} s^{1 / 4}}{(-\ln \varepsilon)^{2}} c^{*}+\cdots,
$$

where the first $\cdots$ represents the higher order terms in the expansion of $4 \tilde{t}$ in terms of $\varepsilon$ and $s$, and the quantity $c^{*}$ is as yet unknown (with $c^{*}=-32 \bar{c}_{1}$ in the previous regime). The temporal multi-scaling incorporates the scale associated with the frequency of the wave outside the slug and also the scale associated with the variation of the basic flow:

$$
\frac{\partial}{\partial \widetilde{t}}=-\widetilde{\alpha} c \frac{\partial}{\partial \widetilde{\xi}}+\left((-\ln \varepsilon)^{2} s+\cdots\right) \frac{\partial}{\partial s},
$$

with $\widetilde{\alpha}, c$ given in (5.2), (5.3). The scalings for the velocity components and pressure can now be inferred directly from the expansions (4.38), together with (5.1), (5.2) and we find

$$
\begin{gathered}
\widetilde{u}=\frac{4}{\mathcal{L}}+\frac{4 \ln s}{\mathcal{L}^{2}} \cdots+\frac{\varepsilon^{1 / 4} s^{1 / 4}}{\mathcal{L}^{2}} u_{2}+\cdots+\frac{\varepsilon^{3 / 2} s^{3 / 4}}{\mathcal{L}^{5}} u_{5}+\cdots, \\
\widetilde{v}=\varepsilon^{-5 / 4} \mathcal{L}^{4} s^{1 / 4} v_{2}+\cdots+\mathcal{L} s^{3 / 4} v_{5}+\cdots, \\
\widetilde{p}=\frac{\varepsilon^{1 / 2} s^{1 / 2}}{\mathcal{L}^{4}} Q_{2} \cos \widetilde{\xi}+\cdots+\frac{\varepsilon^{7 / 4} s}{\mathcal{L}^{7}} Q_{5} \sin \widetilde{\xi}+\cdots,
\end{gathered}
$$

where $\mathcal{L} \equiv-\ln \varepsilon$, and the factors involving $s$ have been chosen to scale this variable out of the governing equations. The terms $\left(u_{5}, v_{5}\right)$ are the first to possess odd, even parts respectively about $\widetilde{\xi}=0$. We note that the pressure retains its simple wave form while the velocity components have more complicated $\widetilde{\xi}$ dependence, much as in the nonlinear critical layer considered earlier (section 4.3). From substitution of (5.4) into the RNS equations (4.1) we find that the slug is governed by the nonlinear inviscid problem

$$
\frac{\partial u_{2}}{\partial \widetilde{\xi}}+\frac{\partial v_{2}}{\partial z}+\frac{v_{2}}{z}=0, \quad\left(u_{2}-c^{*}\right) \frac{\partial u_{2}}{\partial \widetilde{\xi}}+v_{2} \frac{\partial u_{2}}{\partial z}=Q_{2} \sin \widetilde{\xi}
$$

cf. (4.15), (4.16), subject to the regularity conditions on the pipe axis:

$$
\frac{\partial u_{2}}{\partial z}=0, v_{2}=0 \text { on } z=0
$$


The outer condition expresses the matching to the core region where the solutions are as given in (4.43) but with $\widetilde{\alpha}$ and $\widetilde{t}$ now in terms of $\varepsilon$ from (5.2), (5.1). The appropriate condition on $u_{2}$ is found to be

$$
u_{2} \sim-32 I_{0}\left(\frac{1}{2} z\right)+\frac{Q_{2} \sqrt{\pi}}{64} z^{1 / 2} \exp \left(-\frac{1}{2} z\right) \cos \widetilde{\xi} \text { as } z \rightarrow \infty .
$$

It will be seen shortly that the solution of (5.5)-(5.7) is not unique and therefore the higher-order terms play a significant role in determining the flow, just as the higher order terms determine the phase shift in the nonlinear critical layer of section 4.3. Denoting the odd part of $u_{5}$ by $u_{5 O}$ and the even part of $v_{5}$ by $v_{5 E}$, we find that these higher order terms are governed by the linear viscous problem

$$
\begin{gathered}
\frac{\partial u_{5 O}}{\partial \widetilde{\xi}}+\frac{\partial v_{5 E}}{\partial z}+\frac{v_{5 E}}{z}=0 \\
\left(u_{2}-c^{*}\right) \frac{\partial u_{5 O}}{\partial \widetilde{\xi}}+v_{2} \frac{\partial u_{5 O}}{\partial z}+u_{5 O} \frac{\partial u_{2}}{\partial \widetilde{\xi}}+v_{5 E} \frac{\partial u_{2}}{\partial z}= \\
-Q_{5} \cos \tilde{\xi}+\frac{1}{s^{3 / 4}}\left(\frac{\partial^{2} u_{2}}{\partial z^{2}}+\frac{1}{z} \frac{\partial u_{2}}{\partial z}-\frac{u_{2}}{4}\right)
\end{gathered}
$$

with the final term on the right hand side of the second equation expressing a contribution from $\partial \widetilde{u} / \partial \tilde{t}$. The boundary conditions are again those of regularity at $z=0$ and the appropriate match to the core region:

$$
\frac{\partial u_{5 O}}{\partial z}=0, v_{5 E}=0 \text { on } z=0 ; u_{5 O} \sim-\frac{Q_{2}}{2 \sqrt{2 \pi}} z^{-3 / 2} \exp \left(\frac{1}{2} z\right) \sin \widetilde{\xi} \text { as } z \rightarrow \infty .
$$

The flow behaviour in the near-wall regions is essentially unchanged except for the new scaling on $\widetilde{\alpha}$. Thus, in the new regime the main problem is to solve the nonlinear slug flow (5.5), (5.6), (5.7), together with the higher-order problem (5.8), (5.9). In particular we seek solutions for $c^{*}\left(Q_{2}\right)$ and anticipate that $c^{*} \rightarrow-32 \bar{c}_{1}$ as $Q_{2} \rightarrow 0$ to effect a match with the previous structure. The solution of this problem is discussed in the next section. A sketch of the mode structure is given in figure $3(\mathrm{~b})$.

\subsection{The method of solution of the nonlinear slug problem}

First we show that the solution of (5.5)-(5.7) is not unique. If we introduce a streamfunction $\psi_{2}$ such that

$$
u_{2}-c^{*}=\frac{1}{z} \frac{\partial \psi_{2}}{\partial z}, \quad v_{2}=-\frac{1}{z} \frac{\partial \psi_{2}}{\partial \widetilde{\xi}},
$$

then in regions of open streamlines we can perform the change of variables $(\widetilde{\xi}, z) \rightarrow$ $\left(\widetilde{\xi}, \psi_{2}\right)$. In terms of these new variables $(5.5 \mathrm{~b})$ becomes

$$
\left.\left(u_{2}-c^{*}\right) \frac{\partial}{\partial \widetilde{\xi}}\right|_{\psi_{2}}\left(u_{2}-c^{*}\right)=Q_{2} \sin \widetilde{\xi}
$$

and can be integrated to yield the result

$$
\frac{1}{2}\left(u_{2}-c^{*}\right)^{2}=g\left(\psi_{2}\right)-Q_{2} \cos \widetilde{\xi}
$$

where $g\left(\psi_{2}\right)$ is arbitrary, except for having to be consistent with the boundary conditions (5.6), (5.7). In addition, differentiation of (5.10) with respect to $z$ gives

$$
\frac{1}{z} \frac{\partial}{\partial z}\left(u_{2}-c^{*}\right)=g^{\prime}\left(\psi_{2}\right)
$$


which can be regarded as a measure of the vorticity. In order to fix the function $g$ and hence determine the solution we impose a viscous secularity condition (Benney \& Bergeron 1969). This can be derived by examining the higher order linear viscous problem (5.8), (5.9). Using (5.10), (5.11) and after some algebra, the left hand side of (5.8b) can be written as

$$
\left.\frac{\partial}{\partial \widetilde{\xi}}\right|_{\psi_{2}}\left(2\left(g\left(\psi_{2}\right)-Q_{2} \cos \widetilde{\xi}\right) \frac{\partial \psi_{5}}{\partial \psi_{2}}-g^{\prime}\left(\psi_{2}\right) \psi_{5}\right),
$$

where $\psi_{5}$ is the streamfunction corresponding to the $\left(u_{5 O}, v_{5 E}\right)$ motion. Thus, upon integration of $(5.8 b)$ with respect to $\widetilde{\xi}$, and imposition of periodicity of $2 \pi$ in $\widetilde{\xi}$ we obtain the constraint

$$
\int_{0}^{2 \pi}\left(\frac{\partial^{2} u_{2}}{\partial z^{2}}+\frac{1}{z} \frac{\partial u_{2}}{\partial z}-\frac{1}{4} u_{2}\right) \mathrm{d} \widetilde{\xi}=0
$$

with the integration carried out at fixed $\psi_{2}$, i.e. along streamlines of the motion. Using (5.10) and (5.11) this condition can be expressed in terms of a differential equation for $q(g) \equiv g^{\prime}\left(\psi_{2}\right)$. This takes the form

$$
\left(\int_{0}^{2 \pi} \mathcal{L}(g, \widetilde{\xi}) z^{2}(g, \widetilde{\xi}) d \widetilde{\xi}\right) q \frac{\mathrm{d} q}{\mathrm{~d} g}-4 \pi q= \pm \frac{1}{4} \int_{0}^{2 \pi} \mathcal{L}(g, \widetilde{\xi}) \mathrm{d} \widetilde{\xi}-\frac{\pi}{2} c^{*}
$$

with

$$
\mathcal{L}(g, \widetilde{\xi}) \equiv \sqrt{2}\left(g-Q_{2} \cos \widetilde{\xi}\right)^{1 / 2} \text { and } \frac{\partial z^{2}}{\partial g}=-\frac{2}{q(g) \mathcal{L}(g, \widetilde{\xi})},
$$

from integration of (5.10), with the \pm signs according to whether $u_{2}-c^{*} \lessgtr 0$. When $g<Q_{2}$ we have a cat's eye region of closed streamlines and uniform vorticity, with $g$ taking the value $Q_{2}$ on the boundary of this region. Assuming that $u_{2}-c^{*}>0$ on $z=0$, the inner boundary of the cat's eye (i.e. the boundary closest to the pipe axis) $z=z_{1}(\widetilde{\xi})$ say, is given, from (5.13), by

$$
\left(z_{1}(\widetilde{\xi})\right)^{2}=\sqrt{2} \int_{g_{0}}^{Q_{2}} \frac{\mathrm{d} g}{q(g)\left(g-Q_{2} \cos \widetilde{\xi}\right)^{1 / 2}},
$$

where $g_{0}\left(>Q_{2}\right)$ is the unknown value of $g$ that corresponds to the pipe axis $z=0$. Similarly the outer boundary of the cat's eye $z=z_{2}(\widetilde{\xi})$ is found from

$$
\left(z_{2}(\widetilde{\xi})\right)^{2}=\left(z_{\infty}(\widetilde{\xi})\right)^{2}-\sqrt{2} \int_{g_{\infty}}^{Q_{2}} \frac{\mathrm{d} g}{q(g)\left(g-Q_{2} \cos \widetilde{\xi}\right)^{1 / 2}}
$$

where $g_{\infty}$ is an arbitrarily large value of $g$ with $z_{\infty}$ the corresponding value of $z$. The computational procedure to solve the slug problem is as follows.

We first consider the solution in the region $z>z_{2}$ (i.e. between the cat's eye and the pipe wall). Here $u_{2}-c^{*}<0$ and $q<0$. We begin by fixing the value of $Q_{2}$ and making a guess for the corresponding value of the wavespeed correction $c^{*}$. Next, a suitably large value for $g, g_{\infty}$ is chosen, from which a corresponding $u_{2 \infty}(\widetilde{\xi})$ can be derived from (5.10). The value of $z_{\infty}(\widetilde{\xi})$ is then found by applying the outer boundary condition (5.7), which is solved implicitly, and the value of $q_{\infty}$ is found from

$$
q_{\infty}=\frac{1}{z_{\infty}}\left(\frac{\partial u_{2}}{\partial z}\right)_{z=z_{\infty}}
$$

with the right hand side obtained by differentiating (5.7) with respect to $z$ and then setting $z=z_{\infty}$. Equation (5.12) (with a plus sign on the right hand side since $u_{2}-c^{*}<0$ ) 
is marched inwards towards the cat's eye from $g=g_{\infty}$ where $z=z_{\infty}(\widetilde{\xi})$ and $q=q_{\infty}$, and the solution for $q(g)$ is obtained over the range $Q_{2} \leq g \leq g_{\infty}$. The integral in (5.12) is evaluated using Simpson's rule. From the solution, the outer boundary of the cat's eye $z=z_{2}(\widetilde{\xi})$ can be deduced using (5.15), and the value of $q\left(Q_{2}\right), q_{2}$ say, can be calculated. In the second part of the calculation we solve in the region between the cat's eye and the pipe axis, i.e. for $0 \leq z \leq z_{1}(\widetilde{\xi})$. Here we solve (5.12) (with a minus sign on the right hand side since $u_{2}-c^{*}>0$ ) in the range $Q_{2} \leq g \leq g_{0}$ with $g_{0}$ unknown (and guessed initially). Since the coefficient of $q \mathrm{~d} q / \mathrm{d} g$ in (5.12) is zero at $g=g_{0}$, a series solution for $q$ is developed close to this location, and this is used to provide the boundary condition at $g=g_{0}-\delta$, with $0<\delta \ll 1$. The differential equation (5.12) is marched from $g=g_{0}$ away from the pipe axis and towards the cat's eye. The inner boundary of the cat's eye $z=z_{1}(\widetilde{\xi})$ is calculated from $(5.14)$ and the value of the vorticity at the inner edge of the eye, $q\left(Q_{2}\right)=q_{1}$, say, is determined. In order that the cat's eye is closed we require

$$
z_{1}(0)=z_{2}(0)
$$

and in order that the vorticity is continuous across the eye (a consequence of the PrandtlBatchelor theorem) we must have

$$
q_{1}=q_{2} \text {. }
$$

These two conditions are satisfied by iterating upon the values of $c^{*}$ and $g_{0}$ for given $Q_{2}$.

The above procedure assumes that the centre of the cat's eye is offset from the pipe axis, as would be expected at small amplitudes, given the earlier analysis of section 4.3. If the cat's eye is centered at $z=0$ then only the first part of the above procedure is necessary and the value of $c^{*}$ is iterated upon to ensure that $z_{2}(0)=0$.

Solutions can be found for all values of $Q_{2}$ and the calculated values for $c^{*}\left(Q_{2}\right)$ prove to be relatively insensitive to the choice of $g_{\infty}$ which was taken equal to $10^{4}$ for most of the results described here. Results are shown in figures 4-6, and discussed in the next subsection.

\subsection{Numerical results}

We start by considering values of $Q_{2}$ in the range $0<Q_{2}<0.05$ for which the numerical calculations indicate that the centre of the cat's eye is off the pipe axis. In figure $4(\mathrm{a})$ we plot $c^{*}$ versus $Q_{2}$. From (5.3) we see that $c^{*}$ gives a measure of the velocity deficit between the slug propagation speed and the speed of the basic flow on the axis (where it assumes a maximum). We can see that there is a rapid increase in $c^{*}$ from its nonlinear critical layer value as the amplitude $Q_{2}$ is increased: this means that the slug propagates at a speed ever closer to the maximum fluid speed. In figure 4(b) we plot the radial location of the centre of the slug $z_{c}$ versus $Q_{2}$ with $z_{c}=0$ corresponding to the pipe axis. Once again there is a rapid adjustment of the position of the cat's eye as $Q_{2}$ is increased and it is evident that as the nonlinearity of the slug flow is increased, the slug itself moves closer to the pipe axis. These trends are seen clearly in the sequence of figures $5(\mathrm{a})-(\mathrm{c})$ which show the streamlines within the slug region for different values of $Q_{2}$. We also see that the cat's eye region of uniform vorticity (inside which the streamlines are closed) increases in radial extent as it approaches the axis. Beyond the value $Q_{2} \simeq 0.05$, the slug region becomes centered on the pipe axis. Figure $6(\mathrm{a})$ shows how $c^{*}$ varies as $Q_{2}$ is increased further. It is evident that $c^{*}$ is approaching zero, indicating that the nonlinear slug is propagating at virtually the maximum fluid velocity. It becomes increasingly difficult to compute accurate solutions at large values of $Q_{2}$ using the present numerical method, primarily because of difficulties in imposing the outer boundary condition (5.7) at sufficiently large $z_{\infty}$. In figure $6(\mathrm{~b})$-(d) we show streamline plots for $Q_{2}>0.05$. The 


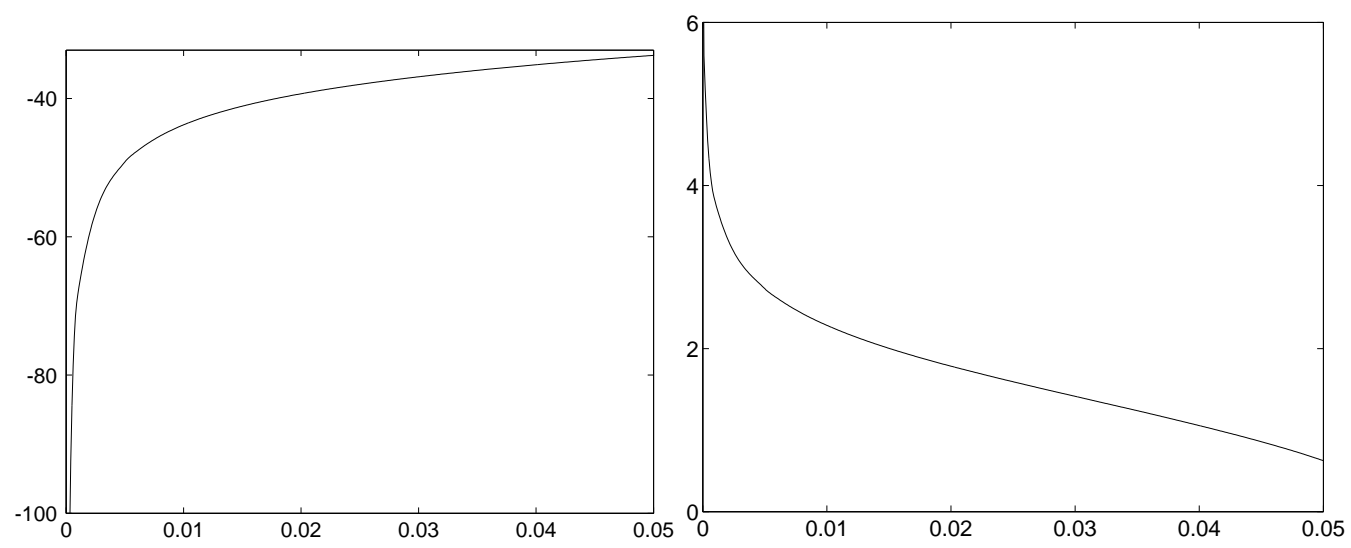

FigURE 4. Solutions of the nonlinear slug problem of section 5. (a) Wavespeed correction $c^{*}$ versus scaled amplitude $Q_{2}$; (b) location of the centre of the cat's eye region $z_{c}$ versus $Q_{2}$.
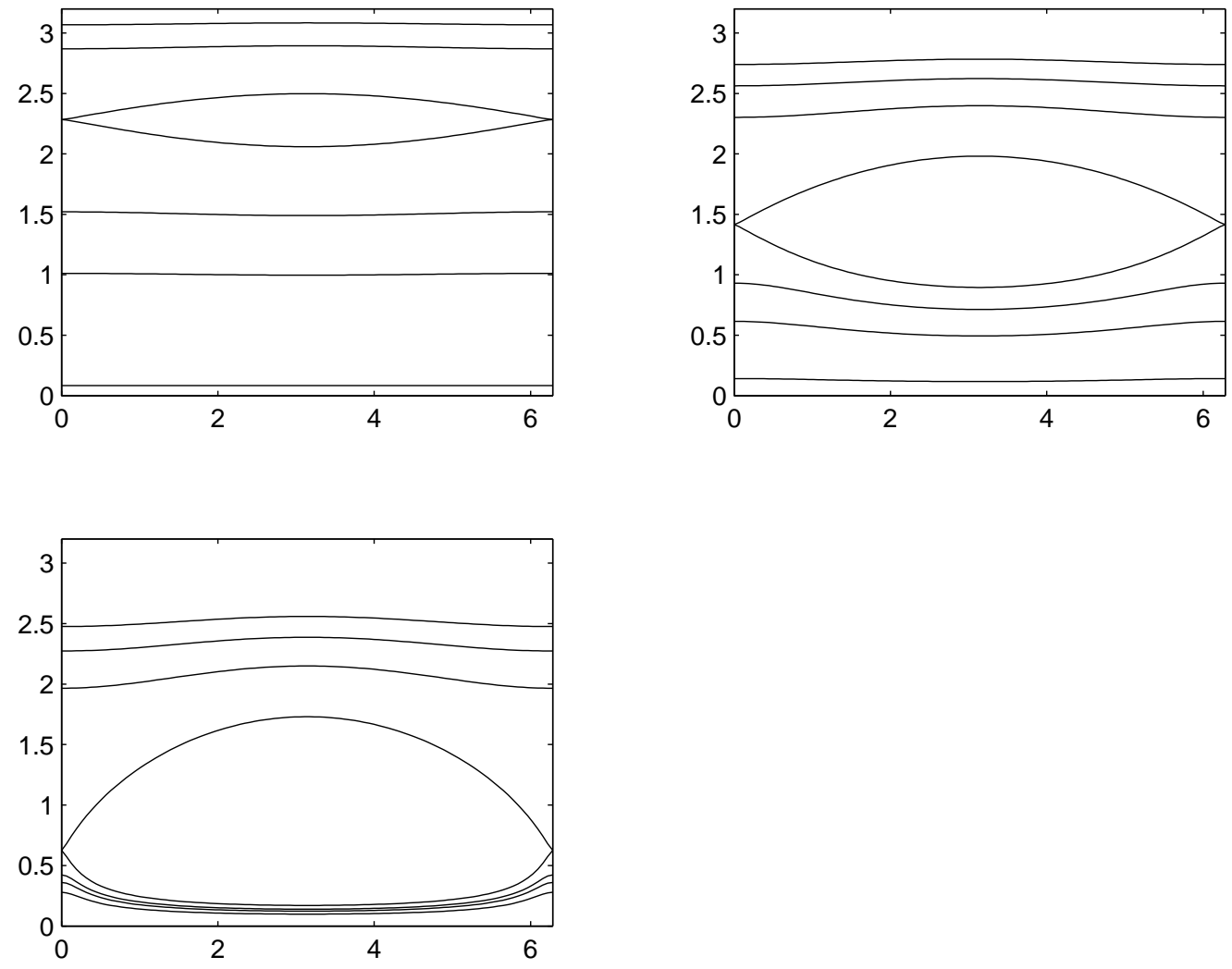

FiguRE 5. Solutions of the nonlinear slug problem. Streamline patterns, corresponding to constant values of $g$, in the slug region for (a) $Q_{2}=0.01$; (b) $Q_{2}=0.03$; (c) $Q_{2}=0.05$. Vertical axis: $z$, horizontal axis: $\widetilde{\xi}$. In each figure the closed contours correspond to the value $g=Q_{2}$.

slug remains centered on the pipe axis but the region of uniform vorticity expands as $Q_{2}$ is increased. 

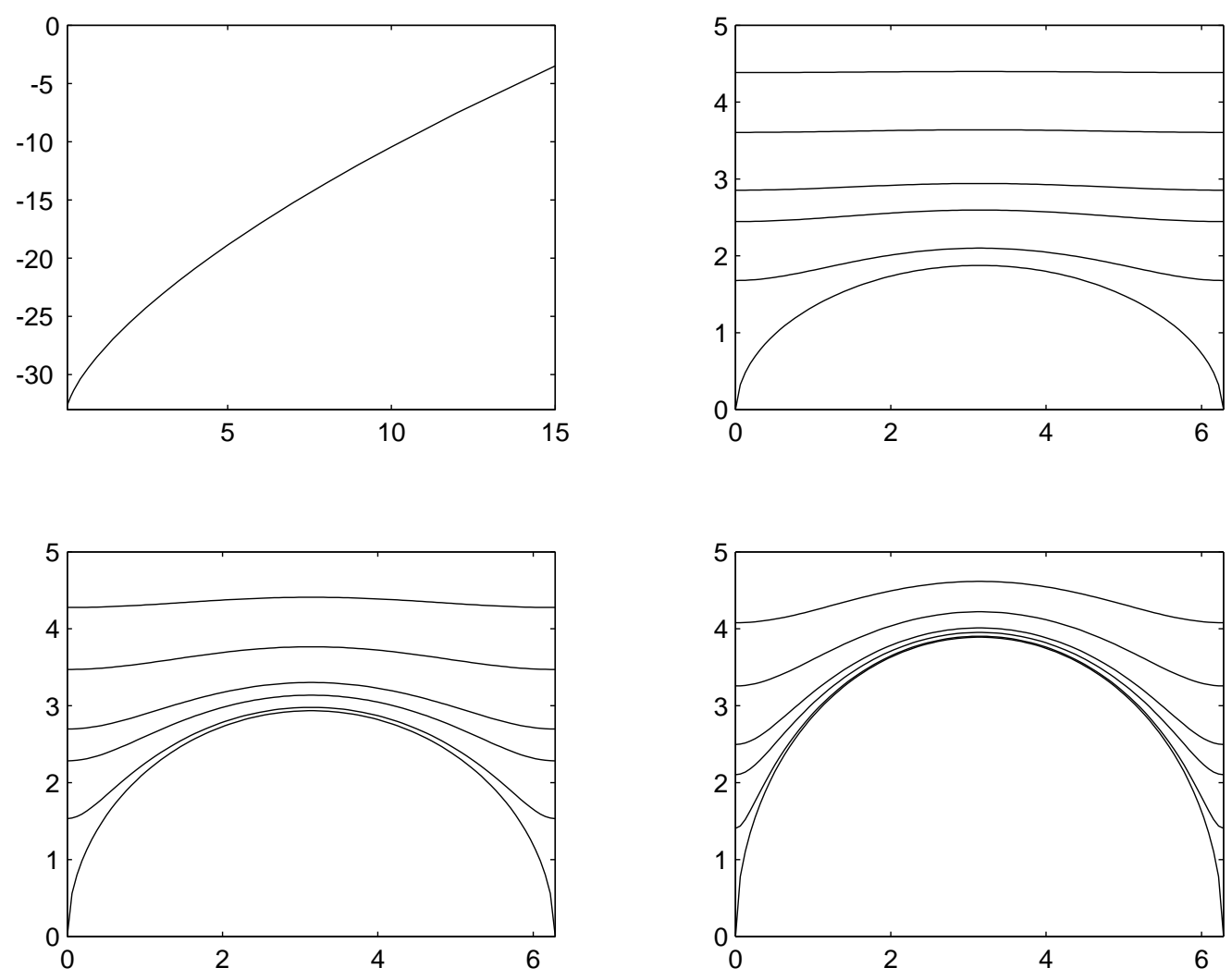

FIGURE 6. Solutions of the slug problem for larger amplitudes, when the cat's eye region is centered on the pipe axis. (a) Wavespeed correction $c^{*}$ versus amplitude $Q_{2}$; (b)-(d) streamline patterns (corresponding to constant values of $g$ ) for: (b) $Q_{2}=0.1$; (c) $Q_{2}=1$; (d) $Q_{2}=5$. Vertical axis: $z$, horizontal axis $\widetilde{\xi}$. In each contour plot the innermost curve corresponds to the value $g=Q_{2}$.

\section{Discussion}

We have shown that when $\tilde{t} \ll 1$, impulsively-started pipe flow supports linear axisymmetric neutral waves of an upper-branch form in which the near-wall critical layer and the viscous wall layer are distinct. When $\widetilde{t}$ is slightly larger, but still small, weakly nonlinear effects enter the critical layer for disturbances with pressure amplitude of order $(\alpha R)^{-1 / 3} \widetilde{t}^{13 / 6}$, with $\alpha R \gg 1$, but the critical layer remains close to the wall and hence the waves propagate slowly relative to the basic flow. In addition, a second strongly nonlinear wave structure, incorporating a region of uniform vorticity on or close to the pipe axis, is also found to exist. For this structure the amplitude scaling is slightly larger, specifically of order $(\alpha R)^{-1 / 3} \widetilde{t}^{5 / 3}$, and the waves propagate at close to the maximum fluid velocity. At $O(1)$ values of $\tilde{t}$ these two distinct structures merge onto one scaling with pressure amplitude $O(\alpha R)^{-1 / 3}$ and an $O(1)$ wavespeed. The new nonlinear structure which emerges is an axisymmetric version of the Smith \& Bodonyi (1982) nonlinear neutral wave structure for HPF. In our structure, for $\widetilde{t}<\widetilde{t}_{c}(\simeq 0.057)$, there are two possible modes, branches I and II, (corresponding to the continuation of the two solutions mentioned above). For both modes the critical layer is sited an $O(1)$ distance from the wall and the value of the wavespeed is calculated from the integral criterion (4.11), rather than from the numerical solution of a Rayleigh equation as in Smith \& Bodonyi 
(1982), Walton (2002). The planar equivalent of this integral condition has arisen before in studies of the finite-time breakdown of interactive boundary layers (e.g. Smith 1988, Li, Walker, Bowles \& Smith 1998), and is regarded by those authors as providing a criterion for the onset of transition in such flows. Since solutions only exist up to a cut-off time $\widetilde{t}_{c}$, the fully-developed HPF does not support axisymmetric disturbances of this particular form.

The most surprising and significant result of the work described in this paper was the discovery of the branch II solution which is clearly a form of neutral centre mode. This mode remains nonlinear even as $\widetilde{t} \rightarrow 0$, and the asymptotic structure is dominated by the presence of a thin region centered on the pipe axis in which the dynamics are governed by an unsteady nonlinear Euler-type problem. A similar asymptotic structure was proposed tentatively by Smith, Doorly \& Rothmayer (1990) as a large amplitude limit of the Smith \& Bodonyi neutral wave solution for HPF. In their case the near-axis flow is governed by the full unsteady three-dimensional Euler equations and no solutions were attempted. In our case, simplifications arise due to (a) the assumed axisymmetric nature of the disturbances, and (b) the long axial lengthscale considered, which means that the underlying governing equations are the RNS equations (4.1). Taken together, these simplifications lead to a system (5.5)-(5.9), which can be integrated and then solved numerically, as described in section 5.1, in a relatively simple way. The results of these calculations show that as $\tilde{t}$ is made progressively smaller, a cat's eye region of uniform vorticity (originally present within the nonlinear critical layer at $O(1)$ values of $\widetilde{t}$ ) moves towards the pipe axis and then retains its position as $\widetilde{t}$ is decreased further. This region of concentrated vorticity is convected downstream at almost the maximum fluid velocity and it is this property that led Smith, Doorly \& Rothmayer to refer to this solution as a 'nonlinear slug flow'. From our numerical solutions of the slug flow, we have seen that the cat's eye region thickens and moves towards the pipe centre as the disturbance amplitude $Q_{2}$ is increased, remaining there beyond a critical value of $Q_{2}$.

Ongoing research suggests that the formation of this type of slug region can be seen theoretically in other related flows such as the unsteady axial flow between sliding, concentric cylinders. In that particular case, the critical layer moves towards the inner pipe wall as time is increased. A more complicated version of the structure also appears to be present in unsteady pipe flow subjected to nonsymmetric disturbances.

Returning now to the case of developing axisymmetric pipe flow, we have established in this paper that as soon as the fluid is set into motion the flow is susceptible to nonlinear axisymmetric travelling-wave disturbances of relatively long wavelength, propagating at speeds close to the centreline velocity, and carrying downstream thin slug-like regions of uniform vorticity. Although concentrated along the pipe axis, the slug is linked to properties near the pipe wall by means of viscous effects acting at higher order (see equation (5.8)). Eventually this 'slug region' moves away from the pipe axis, and finally, beyond an $O(1)$ value of $\widetilde{t}$, neutral waves are no longer supported. Despite this eventual stabilization of the flow, it is possible that these slug solutions play a significant role in pipe flow transition, given that the equilibrium slug itself is likely to be unstable. In fact it is almost certain that all the neutral wave structures calculated in this paper represent unstable equilibrium states, given that the Smith-Bodonyi structure has been shown to be unstable (Walton 2004). The fact that similar slugs of vorticity have been observed in the experiments mentioned in the introduction is encouraging, but it would be going too far to suggest that the structures found here are one and the same. What we have shown is that asymptotic analysis can identify structures which could be the precursors to the puffs and slugs seen experimentally. The long-wavelength structures described here emerge from the wall layer as the disturbance amplitude is increased, eventually becoming 
concentrated along the pipe axis. Of course, many features of puffs and slugs are not present in these relatively simple structures: we cannot, for example, explain their sharp front and trailing interfaces, nor their dependence upon Reynolds number. Although in some ways the idealized assumption of a spatially developed, unsteady flow subject to axisymmetric input is made in order that a tractable problem can be formulated, we feel that we have demonstrated that slug and puff-like structures can arise as a result of problems governed predominantly by inviscid dynamics, but that viscous effects cannot be entirely neglected when attempting to model these phenomena.

The comments of the referees are acknowledged gratefully.

\section{Appendix. Evaluation of the finite part integral criteria}

(i) In section 4 we require the solution $c_{0}(\widetilde{t})$ of

$$
I_{1} \equiv \mathrm{FP} \int_{0}^{1} \frac{r \mathrm{~d} r}{\left(u_{0}(r, \widetilde{t})-c_{0}\right)^{2}}=0,
$$

where $u_{0}$ is the basic flow (1.3) and $c_{0}$ is the value of $u_{0}$ corresponding to the critical level $r=r_{c}(\widetilde{t})$, with $0<r_{c}<1$.

In order to evaluate the integral in (A1) we first rewrite the integrand in the form

$$
\frac{1}{\left(r-r_{c}\right)^{2}} \frac{r\left(r-r_{c}\right)^{2}}{\left(u_{0}-c_{0}\right)^{2}}
$$

and then integrate by parts to obtain

$$
I_{1}=\left[-\frac{r\left(r-r_{c}\right)}{\left(u_{0}-c_{0}\right)^{2}}\right]_{0}^{1}+\mathrm{FP} \int_{0}^{1} \frac{1}{\left(r-r_{c}\right)} \frac{\partial}{\partial r}\left(\frac{r\left(r-r_{c}\right)^{2}}{\left(u_{0}-c_{0}\right)^{2}}\right) \mathrm{d} r
$$

Applying the no-slip condition $u_{0}=0$ when $r=1$ and integrating by parts a second time, we find

$$
\begin{array}{r}
I_{1}=-\frac{\left(1-r_{c}\right)}{c_{0}^{2}}+\left(\frac{2}{c_{0}^{3}}\left(1-r_{c}\right)^{2} \frac{\partial u_{0}}{\partial r}(1, \widetilde{t})+\frac{2\left(1-r_{c}\right)+\left(1-r_{c}\right)^{2}}{c_{0}^{2}}\right) \ln \left(1-r_{c}\right) \\
-\frac{r_{c}^{2} \ln r_{c}}{\left(u_{0}(0, \widetilde{t})-c_{0}\right)^{2}}-\int_{0}^{1} \frac{\partial^{2}}{\partial r^{2}}\left(\frac{r\left(r-r_{c}\right)^{2}}{\left(u_{0}-c_{0}\right)^{2}}\right) \ln \left|r-r_{c}\right| \mathrm{d} r \\
=0 .
\end{array}
$$

The integral in (A2) is well-defined and can be easily evaluated numerically, for given $c_{0}$, using a package such as Mathematica. Typically, 100 terms were found to be sufficient in the series representation for $u_{0}$ in (1.3). We solve (A2) for given $\widetilde{t}$ by first guessing a value for $r_{c}$ (with $c_{0}$ following from $c_{0}=u_{0}\left(r_{c}, \widetilde{t}\right)$ ), and then using Newton iteration to update $r_{c}$ until $I_{1}=0$ to a prescribed tolerance. Use of this procedure leads to the graph of $r_{c}(\widetilde{t})$ shown in figure $2(\mathrm{a})$, with the wave amplitude $A_{0}(\widetilde{t})$ (shown in figure $2(\mathrm{~b})$ ) then following from (4.28).

(ii) In section 4.5 we require the solution $z_{c}$ such that

$$
I_{2}=\mathrm{FP} \int_{0}^{\infty} \frac{z \mathrm{~d} z}{\left(I_{0}\left(\frac{1}{2} z\right)-I_{0}\left(\frac{1}{2} z_{c}\right)\right)^{2}}=0,
$$

where $I_{0}$ is a modified Bessel function and $\bar{c}_{1}=I_{0}\left(\frac{1}{2} z_{c}\right)$. 
The method of evaluation follows closely that described in (i), and after integrating by parts twice we obtain

$$
I_{2}=-\frac{z_{c}^{2} \ln z_{c}}{\left(1-I_{0}\left(\frac{1}{2} z_{c}\right)\right)^{2}}-\int_{0}^{\infty} \frac{d^{2}}{d z^{2}}\left(\frac{z\left(z-z_{c}\right)^{2}}{\left(I_{0}\left(\frac{1}{2} z\right)-I_{0}\left(\frac{1}{2} z_{c}\right)\right)^{2}}\right) \ln \left|z-z_{c}\right| \mathrm{d} z=0,
$$

where all other integrated contributions are zero. Once again, Newton iteration is used to iterate upon $z_{c}$ until $I_{2}=0$. We find that to three decimal places the value of $z_{c}$ is given by $z_{c} \simeq 8.281$, as quoted in section 4.5 .

\section{REFERENCES}

Bandyopadhyay, P. R. 1986 Aspects of the equilibrium puff in transitional pipe flow. J. Fluid Mech. 163, 439-458.

Bandyopadhyay, P. R. \& Walton, A. G. 1990 Perturbation amplification in the entry region of a transitional pipe flow. In Instability and Transition, Volume 1 (ed. M. Y. Hussaini \& R. G. Voigt), pp. 355-371. Springer-Verlag.

Batchelor, G. K. 1956 On steady laminar flow with closed streamlines at large Reynolds number. J. Fluid Mech. 1, 177-190.

Batchelor, G. K. 1967 An Introduction to Fluid Dynamics. Cambridge University Press.

Benney, D. J. \& Bergeron, R. F. 1969 A new class of nonlinear waves in parallel flows. Stud. Appl. Maths 48, 181-204.

Darbyshire, A. G. \& Mullin, T. 1995 Transition to turbulence in constant-mass-flux pipe flow. J. Fluid Mech. 289, 83-114.

Duguet, Y., Willis, A. P. \& Kerswell, R. R. 2010 Slug genesis in cylindrical pipe flow. To appear in J. Fluid Mech.

Faisst, H. \& Eckhardt, B. 2003 Traveling waves in pipe flow. Phys. Rev. Lett. 91, 224502.

Fowler, A. C. \& Howell, P. D. 2003 Intermittency in the transition to turbulence. SIAM J. Appl. Math. 63, 1184-1207.

Fox, J. A., Lessen, M. \& Bhat, W. V. 1968 Experimental investigation of the stability of HagenPoiseuille flow. Phys. Fluids 11, 1-4.

Huang, L. M. \& Chen, T. S. 1974a Stability of developing pipe flow subjected to nonaxisymmetric disturbances. J. Fluid Mech. 63, 183-193.

Huang, L. M. \& Chen, T. S. 1974b Stability of the developing laminar pipe flow. Phys. Fluids 17, 245-247.

Li, L., Walker, J. D. A., Bowles, R. I. \& Smith, F. T. 1998 Short-scale break-up in unsteady interactive layers: local development of normal pressure gradients and vortex wind-up. $J$. Fluid Mech. 374, 335-378.

Leite, R. J. 1959 An experimental investigation of the stability of Poiseuille flow. J. Fluid Mech. 5, 81-96.

Lin, C. C. 1955 The Theory of Hydrodynamic Stability. Cambridge University Press.

Nishi, M., Unsal, B., Durst, F. \& Biswas, G. 2008 Laminar-to-turbulent transition of pipe flows through puffs and slugs. J. Fluid Mech. 614, 425-446.

O'Sullivan, P. L. \& Breuer, K. S. 1994 Transient Growth in circular pipe flow. II. Nonlinear development. Phys. Fluids 6, 3652-3664.

Priymak, V. G. \& Miyazaki, T. 2004 Direct numerical simulation of equilibrium spatially localized structures in pipe flow. Phys. Fluids 16, 4221-4234.

Reid, W. H. 1965 The stability of parallel flows. In Basic Developments in Fluid Dynamics, Vol. 1 (ed. M. Holt). Academic Press.

Reynolds, O. 1883 An experimental investigation of the circumstances which determine whether the motion of water will be direct or sinuous, and of the law of resistance in parallel channels. Phil. Trans. R. Soc. Lond. A 174, 935-982.

Salwen, H., Cotton, F. W. \& Grosch, C. E. 1980 Linear stability of Poiseuille flow in a circular pipe. J. Fluid Mech. 98, 273-284.

Sarpkaya, T. 1975 A note on the stability of developing laminar pipe flow subjected to axisymmetric and non-axisymmetric disturbances. J. Fluid Mech. 68, 345-351. 
Schmid, P. J. \& Henningson, D. S. 2000 Stability and transition in Shear Flows. Springer.

Shan, H., Ma, B., Zhang, Z. \& Nieuwstadt, F. T. M. 1999 Direct numerical simulation of a puff and a slug in transitional cylindrical pipe flow. J. Fluid Mech. 387, 39-60.

Smith, F. T. 1988 Finite-time break-up can occur in any unsteady interacting boundary layer. Mathematika, 35, 256-273.

Smith, F. T. \& Bodonyi, R. J. 1982 Amplitude-dependent neutral modes in the Hagen-Poiseuille flow through a circular pipe. Proc. R. Soc. Lond. A 384, 463-489.

Smith, F. T., Doorly, D. J. \& Rothmayer, A. P. 1990 On displacement-thickness, wall-layer and mid-flow scales in turbulent boundary layers, and slugs of vorticity in channel and pipe flows. Proc R. Soc. Lond. A 428, 255-281.

Stuart, J. T. 1963 Hydrodynamic stability. In Laminar Boundary Layers (ed. L. Rosenhead). Dover.

Szymanski, P.1932 Quelques solutions exactes des equations de l'hydrodynamique du fluide visqeux dans le cas d'un tube cylindrique. J. Math. Pure Appl. Series 9, 11, 67-107.

Tatsumi, T. 1952a Stability of the laminar inlet-flow prior to the formation of Poiseuille regime, I. J . Phys. Soc. Japan 7, 489-495.

Tatsumi, T. 1952b Stability of the laminar inlet-flow prior to the formation of Poiseuille regime, II. J . Phys. Soc. Japan 7, 495-502.

van Doorne, C. W. H. \& Westerweel, J. 2009 The flow structure of a puff. Phil. Trans. R. Soc. Lond. A 367, 489-507.

Walton, A. G. 2002 The temporal evolution of neutral modes in the impulsively started flow through a circular pipe and their connection to the nonlinear stability of Hagen-Poiseuille flow. J. Fluid Mech. 457, 339-376.

Walton, A. G. 2004 The stability of nonlinear neutral modes in Hagen-Poiseuille flow. Proc. $R$. Soc. Lond. A 461, 813-824.

Wedin, H. \& Kerswell, R. R. 2004 Exact coherent structures in pipe flow: travelling wave solutions. J. Fluid Mech. 508, 333-371.

Wygnanski, I. J. \& Champagne, F. H. 1973 On transition in a pipe. Part 1. The origin of puffs and slugs and the flow in a turbulent slug. J. Fluid Mech. 59, 281-335.

Wygnanski, I. J., Sokolov, M. \& Friedman, D. 1975 On transition in a pipe. Part 2. The equilibrium puff. J. Fluid Mech. 69, 283-304. 